Pacific Journal of Mathematics

CLOSED OPERATORS AND THEIR ADJOINTS ASSOCIATED 


\section{CLOSED OPERATORS AND THEIR ADJOINTS ASSOCIATED WITH ELLIPTIC DIFFERENTIAL OPERATORS}

\section{R. S. FREEMAN}

We are concerned here with determining some closed operators associated with a given elliptic differential operator $A$ of order $2 m$ and some in general nonlocal boundary operators. We seek conditions in particular which guarantee that the result is a normally solvable operator, i.e. with closed graph and closed range in the sense of Visik. We follow basically the method used in Bade and Freeman in the sense that we regard the operator with nonlocal boundary conditions as a perturbation of an operator with boundary conditions defined by a normal set of differential operators $B=$ $\left\{B_{0}, \cdots, B_{m-1}\right\}$ satisfying the condition of Agmon, Douglis and Nirenberg (also Browder, and Schechter). Since the basic a priori estimate valid for such systems essentially says that the resulting operator has closed graph we call such a system $(A, B)$ closable elliptic.

In addition to dealing with higher order elliptic operators and general boundary conditions we also drop the requirement that our region be relatively compact and instead make the weaker requirement that the differential operator in $H^{2 m}(\Omega)$ with local boundary conditions yields an operator with closed range. We work here in $L^{2}$ only and consider operators defined in $H^{2 m}(\Omega)$, in the graph topology associated with the so called maximal operator and in a family of spaces interpolated between these two. Most of our results can be obtained, at least for relatively compact regions, in $L^{p}$ with $1<p<\infty$ at the expense of a somewhat more complicated treatment. A particular complication arises from the fact that different interpolation methods which yield the same spaces in $L^{2}$ do not in general in $L^{p}, p \neq 2$.

The paper is divided into eight sections the first five of which are of a preliminary nature and contain results many of which are variants of well known results.

While in the process of writing this paper we were able to see the thesis of R. W. Beals which he kindly sent to us. The two papers are concerned with similar problems but the results cannot be ordered by inclusion.

1. Preliminaries. As usual points in $R^{n}$ (n-dimensional Euclidean space) are denoted by $x=\left(x_{1}, \cdots, x_{n}\right)$ and $n$-dimensional Lebesgue 
measure by $d x$. We let $d \sigma$ denote $n-1$ dimensional surface measure, $D_{j}=(1 / i)\left(\partial / \partial x_{j}\right)$ where $i^{2}=-1$ and $N$ denote the nonnegative integers. If $\alpha=\left(\alpha_{1}, \cdots, \alpha_{n}\right) \in N^{n}$ and $\xi=\left(\xi_{1}, \cdots, \xi_{n}\right) \in R^{n}$ then $|\alpha|=\sum_{j=1}^{n} \alpha_{j}$, $D^{\alpha}=D_{1}^{\alpha_{1}} \cdots D_{n}^{\alpha_{n}}, \xi^{\alpha}=\xi_{1}^{\alpha_{1}} \cdots \xi_{n}^{\alpha_{n}}$, and $\alpha !=\alpha_{1} ! \cdots \alpha_{n} !$.

If $E$ and $F$ are two topological vector spaces over $C$ the complex numbers we will use the notation $E \subset F$ to mean that $E$ is a subspace of $F$ and that the topology of $E$ is finer than that induced on $E$ by $F$. $\mathscr{S}(E, F)$ will denote the (algebraic) space of continuous linear maps from $E$ to $F$. As is usual $\mathscr{L}(E, C)$ will be denoted by $E^{\prime}$.

Using the notation of L. Schwartz [27] we let $\mathscr{D}(\Omega)$ be the space of infinitely differentiable functions having compact support in $\Omega$ and if $\mathscr{D}(\Omega)$ is equipped with its usual locally convex topology its dual space, the space of distributions on $\Omega$ is denoted by $\mathscr{D}^{\prime}(\Omega)$. In general if $\mathscr{F}(\Omega)$ is a space of functions on $\Omega$ then $\mathscr{F}\left(R^{n}\right)$ is simply denoted by $\mathscr{F} . \mathscr{D}(\bar{\Omega})$ is the space of restrictions to $\Omega$ of functions in $\mathscr{D}$. $\mathscr{S}$ will denote the space of rapidly decreasing functions on $R^{n}$. An element in its dual is called a tempered distribution assuming that $\mathscr{S}$ has its usual locally convex topology.

For $u \in \mathscr{D}(\bar{\Omega})$ and $m \in N$ we let

$$
\|u\|_{m, \Omega}^{2}=\int_{\Omega} \sum_{k=0}^{m}\left(\begin{array}{l}
m \\
k
\end{array}\right) \sum_{|\alpha|=k} \frac{m !}{\alpha !}\left|D^{\alpha} u\right|^{2} d x
$$

suppressing the $\Omega$ when no confusion is likely to result.

When $\Omega$ is $R^{n}$ and if $\hat{u}$ denotes the Fourier-Plancherel transform of $u$ then $\|u\|_{m}^{2}=\int\left(1+|\xi|^{2}\right)^{m}|\widehat{u}(\xi)|^{2} d \xi$ which is the reason for all the factorials. The completion of $\mathscr{D}(\bar{\Omega})$ in the norm $\|\cdot\|_{m}$ is denoted by $H^{m}(\Omega)$ and the completion of $\mathscr{D}(\Omega)$ in the norm $\|\cdot\|_{m}$ is denoted by $H_{0}^{m}(\Omega)$. If $Q$ is $R^{n}$ the two spaces are the same and their elements are tempered distributions.

Let $\Omega$ be an open set in $R^{n}$ whose boundary $\Gamma$ is an infinitely differentiable, orientable manifold. $\Omega$ is not assumed to be relatively compact but is assumed to be uniformly regular in the sense of Browder $[5,6]$. Let $\mathscr{B}^{r}$ be the open unit ball of radius $r$ about the origin. Then we assume there exists a covering of $\Omega$ by open sets $\left\{\Omega_{j}: j \in N\right\}$, a family of infinitely differentiable homeomorphisms $\left\{\varphi_{j}: j=1, \cdots\right\}$ and a positive integer $n_{0}$ such that

(1) At most $n_{0}$ of the $\Omega_{j}$ 's have nonempty intersection.

(2) For $j=1, \cdots \varphi_{j}: \Omega_{j} \cap \Omega \rightarrow \mathscr{B}^{1} \cap\left\{y_{n}>0\right\}$ and $\varphi_{j}: \Omega_{j} \cap \Gamma \rightarrow$ $\mathscr{B}^{\prime} \cap\left\{y_{n}=0\right\}$.

(3) The derivatives of $\varphi_{j}$ and its inverse are uniformly bounded.

(4) $\bigcup_{j} \varphi_{j}^{-1}\left(\mathscr{B}^{\frac{1}{2}}\right)$ covers a uniform neighborhood of $\Gamma$.

It follows [6] that there exists an infinitely differentiable partition of unity $\left\{\eta_{j}: j \in N\right\}$ subordinate to the cover such that in a neighborhood 
of each point $x \in \Gamma$ at most $n_{0}$ of the $\eta_{j}$ 's do not vanish. A number of results usually obtained for compact regions can also be obtained for uniformly regular regions. In an earlier version of this work we included the modifications necessary to prove these results for uniformly regular but not necessarily compact regions. Since that time we have received a copy of the thesis of $\mathrm{R}$. W. Beals in which detailed proofs of these results are given. Consequently we do not duplicate them here. We summarize some well known facts about the spaces $H^{m}(\Omega)$, $m \in N$ in the following

THEOREM 1.1. (i ) If $m, p \in N$ and $m<p$ then $H^{p}(\Omega) \subset H^{m}(\Omega)$ and $H^{p}(\Omega)$ is dense in $H^{m}(\Omega)$.

(ii) $H^{m}(\Omega)$ can be identified with the space of distributions on $\Omega$ all of whose derivatives up to and including order $m$ are functions in $L^{2}(\Omega)=H^{0}(\Omega)$.

(iii) Let $H_{a c}^{m}$ be the closed subspace of $H^{m}$ consisting of those functions in $H^{m}$ whose support is contained in $\Omega^{c}$. Each $u \in H^{m}(\Omega)$ is the restriction to $\Omega$ of $a \tilde{u} \in H^{m}$ and $H^{m}(\Omega)$ is topologically isomorphic to the quotient space $H^{m} / H_{a c}^{m}$ if the latter space is given the quotient topology.

(iv) If $\Omega$ is relatively compact, $m, p \in N$ with $p>m$ then the canonical injection of $H^{p}(\Omega)$ into $H^{m}(\Omega)$ is compact.

A detailed proof of (ii) appears in Browder [6] and a detailed proof of the first statement of (iii) appears in Beals [4] and for $\Omega$ relatively compact in Lions [13].

For $u \in \mathscr{D}(\bar{\Omega})$ we define two more norms

$$
\|u\|_{-m, \Omega}=\sup \left\{\left|(u, v)_{0}\right|: v \in H^{m}(\Omega),\|v\|_{m}=1\right\}
$$

and

$$
\|u\|_{-m}=\sup \left\{\left|(u, v)_{0}\right|: v \in H_{0}^{m}(\Omega),\|v\|_{m}=1\right\} .
$$

The completion of $\mathscr{D}(\bar{\Omega})$ in the first norm is denoted by $H_{\Omega}^{-m}$ and in the second norm by $H^{-m}(\Omega)$. When no confusion is likely to result we suppress the $\Omega$ in the first norm. The space $H^{-m}(\Omega)$ can be identified with a space of distributions on $\Omega$ but since $\mathscr{Q}(\Omega)$ is not dense in $H^{m}(\Omega)$ this is not true for $H_{\bar{\Omega}}^{-m}$. We summarize some well known facts about these spaces. A proof of the last appears in Magenes-Stampacchia [21].

THEOREM 1.2. (i ) $H_{\overline{\bar{\Omega}}}^{-m}\left(H^{-m}(\Omega)\right)$ is topologically isomorphic to the dual space of $H^{m}(\Omega)\left(H_{0}^{m}(\Omega)\right)$.

(ii) If $u \in H^{m}(\Omega)\left(H_{0}^{m}(\Omega)\right)$ and $v \in H_{\overline{\bar{\Omega}}}^{-m}\left(H^{-m}(\Omega)\right)$ the expression 
$(u, v)_{0}$ is well defined and $\left|(u, v)_{0}\right| \leqq\|u\|_{m}\|v\|_{-m, \Omega}\left(\|u\|_{m}\|v\|_{-m}\right)$.

(iii) If $m, p$ are integers, $m>p$ then $H^{-p}(\Omega) \subset H^{-m}(\Omega)$ $\left(H_{\bar{\Omega}}^{-p} \subset H_{\bar{\Omega}}^{-m}\right)$ and $H_{\bar{\Omega}}^{p}$ is dense in $H_{\bar{\Omega}}^{-m}\left(H^{-p}(\Omega)\right.$ is dense in $\left.H^{-m}(\Omega)\right)$.

(iv) If $m \in N$ the space $H_{\bar{\Omega}}^{-m}$ can be identified with the closed subspace of $H^{-m}$ consisting of those elements with support contained in $\bar{\Omega}$.

2. Some interpolation results. There are two methods of interpolating Banach spaces between pairs of Banach spaces which have been widely used, a complex variable method introduced by Calderon [7] and independently by Lions [14] and a real variable method introduced by Lions [15]. Other methods have been discussed for example in Lions-Peetre [20] and in Calderon [7]. We outline briefly the first two mentioned methods here for the convenience of the reader. We summarize below some facts all of which except possibly the last are well known. We do not know whether the last statement appears elsewhere in the literature. Its proof is quite straight forward and we do not include it here.

THEOREM 2.1. Let $H_{0}$ and $H_{1}$ be Hilbert spaces with $H_{1} \subset H_{0}, H_{1}$ being dense in $H_{0}$. Let $\|\cdot\|_{j},(\cdot, \cdot)_{j}, j=0,1$ denote the norm and scalar product in $H_{j}$. Then

(i) There exists a positive-definite, self-adjoint operator $T^{2}$ in $H_{0}$ with domain $D\left(T^{2}\right)=\left\{v \in H_{1}\right.$ : for some $k(v)>0\left|(u, v)_{1}\right| \leqq k(v)\|u\|_{0}$ for all $\left.u \in H_{1}\right\} . D\left(T^{2}\right)$ is dense in $H_{1}$ and for $v \in D\left(T^{2}\right)$ and $u \in H_{1}$, $(u, v)_{1}=\left(u, T^{2} v\right)_{0}$.

(ii) $T$, the positive square root of $T^{2}$, has domain precisely equal to $H_{1}$ and is a continuous bijection of $H_{1}$ onto $H_{0}$. For $u \in H_{1}$, $\|T u\|_{0}=\|u\|_{1}$.

(iii) $T$ has an extension $\widetilde{T}$ to all of $H_{0}$ and $\widetilde{T}$ is a continuous bijection (i.e. an isomorphism) of $H_{0}$ onto a Hilbert space $H_{-1}$ topologically isomorphic to the dual space of $H_{1}$. If for $u \in H_{0}$ we set $\|u\|_{-1}=\left\|T^{-1} u\right\|_{0}$ then $\|u\|_{-1}=\sup \left\{\left|(u, v)_{0}\right|: u \in H_{1}\right.$ and $\left.\|v\|_{1}=1\right\}$.

Since $T$ is also self adjoint, if $0<s<1$, the powers $T^{s}$ of $T$ can be defined with the aid of the spectral measure of $T$ (or of $T^{2}$ ). They are also positive-definite and self-adjoint. We make the

Definition 2.2. For $0<s<1$ let $H_{s}$ be the domain of $T^{s}$ and for $u \in H_{s}$ set $\|u\|_{s}=\left\|T^{s} u\right\|_{0}$. Let, for $u \in H_{0},\|u\|_{-s}=\sup \left\{\left|(u, v)_{0}\right|\right.$ : $v \in H_{s}$ and $\left.\|v\|_{s}=1\right\}$. Denote the completion of $H_{0}$ in the norm $\|\cdot\|_{-s}$ by $H_{-s}$.

Since $T^{s}$ is self-adjoint it is closed and since it has also a continuous inverse it follows that the spaces $H_{s}, 0<s<1$ and Hilbert spaces. 
We summarize some of the salient facts about $H_{s}$ in the following

Theorem 2.3. (i ) For $-1 \leqq s \leqq 1, H_{s}$ is a Hilbert space.

(ii) For $-1 \leqq s<t \leqq 1, H_{t} \subset H_{s}, H_{t}$ being dense in $H_{s}$.

(iii) The map $T^{s}$ has an extension $\left(T^{s}\right)^{2}=(\widetilde{T})^{s}$ to $H_{0}$ and yields an isomorphism of $H_{0}$ onto $H_{-s}$. $H_{-s}$ is isomorphic to the dual space of $H_{s}$.

(iv) Let $H_{0}=V_{0}$ and $H_{s}=V_{1}$ for some $0<s<1$. Construct the space $V_{\theta}$, for $0<\theta<1$, as above. Then $V_{\theta}=H_{\theta s}$.

The last mentioned fact follows easily from the manner in which the spaces have been constructed and the fact that a self adjoint operator can have no proper self adjoint extension.

We give now a definition due (with a slight modification in notation) to Lions [14].

DeFinition 2.4. Let $\mathscr{F}\left(H_{2 s}, H_{0}, R_{+}\right)$be the set of all strongly Lebesgue measurable $H_{1}$ valued functions on $R_{+}=[0, \infty)$ such that $\int_{0}^{\infty}\|u(t)\|_{2 s}^{2} d t$ and $\int_{0}^{\infty}\left\|u^{\prime}(t)\right\|_{0}^{2} d t<\infty$.

Theorem [Lions] 2.5. For $u \in \mathscr{F}\left(H_{2 s}, H_{0}, R_{+}\right)$the map $u \rightarrow u(0)$ can be defined and is a continuous map of $\mathscr{F}\left(H_{2 s}, H_{0}, R_{+}\right)$onto $H_{s}$.

Let $V_{1}$ and $V_{0}$ be another pair of Hilbert spaces with $V_{1}$ dense in $V_{0}, V_{1} \subset V_{0}$ and construct for $0<s<1$ the spaces $V_{s}$. Then

Theorem [Lions] 2.6. If $L \in \mathscr{L}\left(H_{0}, V_{0}\right)$ and in $\mathscr{L}\left(H_{1}, V_{1}\right)$ then $L \in \mathscr{S}\left(H_{s}, V_{s}\right)$ for $0<s<1$.

The spaces $H_{s}, 0<s<1$ will be called trace spaces.

Now suppose $A_{0}$ and $A_{1}$ are two Banach spaces each continuously embedded in some topological vector space $\mathscr{A}$. Equip $A_{0}+A_{1}$ with the norm $\|a\|_{A_{0}+A_{1}}=\inf \left(\left\|a_{0}\right\|_{A_{0}}+\left\|a_{1}\right\|_{A_{1}}: a=a_{0}+a_{1}\right) . \quad A_{0}+A_{1}$ is then a Banach space. Let $\mathscr{H}\left(A_{0}, A_{1}\right)$ be the set of maps of the strip $0 \leqq$ $\operatorname{Re} z \leqq 1$ into $A_{0}+A_{1}$, holomorphic in $0<\operatorname{Re} z<1$ and continuous and bounded in $0 \leqq \operatorname{Re} z \leqq 1$. Suppose moreover that $f(i y) \in A_{0}, f(1+i y) \in A_{1}$ and as $|y| \rightarrow \infty\|f(i y)\|_{A_{0}} \rightarrow 0$ and $\|f(1+i y)\|_{A_{1}} \rightarrow 0$. Equip $\mathscr{H}\left(A_{0}, A_{1}\right)$ with the norm $\|f\|_{\mathscr{C}\left(A_{0}, A_{1}\right)}=\max \left(\sup _{y}\|f(i y)\|_{A_{0}}\right.$, $\left.\sup _{y}\|f(1+i y)\|_{A_{1}}\right)$. Let $\left[A_{0}, A_{1}, \delta(\theta)\right]$ for $0 \leqq \theta \leqq 1$ be the image in $A_{0}+A_{1}$ of the map $\theta \rightarrow f(\theta)$ and equip $\left[A_{0}, A_{1}, \delta(\theta)\right]$ with the norm $\|a\|_{\theta}=\inf \left\{\|f\|_{\mathscr{K}\left(A_{0}, A_{1}\right)}\right.$ : $f(\theta)=a\} . \quad\left[A_{0}, A_{1}, \delta(\theta)\right]$ is then a Banach space.

Suppose $B_{0}$ and $B_{1}$ is another pair of Banach spaces each continuously embedded in a topological vector space $\mathscr{B}$. Then 
Theorem (Lions [14], Calderon [7]) 2.7. If $T \in \mathscr{L}\left(A_{0}, B_{0}\right)$ and $T \in \mathscr{S}\left(A_{1}, B_{1}\right)$ then for $0<\theta<1, T \in \mathscr{L}\left(\left[A_{0}, A_{1}, \delta(\theta)\right],\left[B_{0}, B_{1}, \delta(\theta)\right]\right)$.

Let $\mu$ be a finite measure on $R^{+}$and $H_{0}=\mathscr{L}^{2}\left[R_{+}, d \mu\right], H_{1}=$ $\mathscr{L}^{2}\left[R_{+}, \lambda d \mu\right]$ then it is easy to see that $\left[H_{0}, H_{1}, \delta(\theta)\right]$ is $L^{2}\left[R_{+}, \lambda^{\theta} d \mu\right]$. If $H_{0}$ and $H_{1}$ are Hilbert spaces, $H_{1} \subset H_{0}$ and $H_{1}$ dense in $H_{0}$ and if $\mu$ is the spectral measure of the self adjoint operator $T$ of Theorem 2.1 then there is a unitary map $U$ mapping $H_{1}$ onto $L^{2}\left[R_{+}, \lambda d \mu\right]$ and $H_{0}$ onto $L^{2}\left[R_{+}, d \mu\right]$. Thus

THEOREM 2.8. If $H_{1}, H_{0}$ is a pair of Hilbert spaces with $H_{1} \subset H_{0}$ and $H_{1}$ dense in $H_{0}$, then for $0<\theta<1,\left[H_{0}, H_{1}, \delta(\theta)\right]=H_{\theta}$.

Definition 2.9. If $s$ is a real number $m \leqq s \leqq m+1$ we let $H^{s}(\Omega)=\left[H^{m}(\Omega), H^{m+1}(\Omega), \delta(s-m)\right]$.

THEOREM 2.10. $H^{s}(\Omega)$ for $s$ real, $s \geqq 0$, is isomorphic to the space of restrictions to $\Omega$ of elements of $H^{s}\left(R^{n}\right)$.

REMARK 2.11. If $0 \leqq s \leqq m$ we could also define $H^{s}(\Omega)$ by interpolating between $H^{0}(\Omega)$ and $H^{m}(\Omega)$ using Theorem 2.1. By Theorem 1.1, part iii, and Theorems 2.6 and 2.7 the spaces are the same. The operator $T^{2}$ of Theorem 2.1 is the operator $(I+\Delta)^{m}$ subject to the Neumann boundary condition (homogeneous), where $\Delta=\sum_{j=1}^{n} D_{j}^{2}$. If $m=2 p$, the operator $(I+\Delta)^{p}$ with domain $H^{p}(\Omega)$ is not even closed, much less self adjoint. Thus the square root of $(I+4)^{2 p}$ with the homogeneous, Neumann boundary conditions does not look like $(I+\Delta)^{p}$.

Lemma 2.12. Given $\varepsilon>0$ there exists a $C(\varepsilon)>0$ such that for $0 \leqq s<t$ and $u \in \mathscr{D}(\bar{\Omega})$

$$
\|u\|_{s} \leqq \varepsilon\|u\|_{t}+C(\varepsilon)\|u\|_{0} \text {. }
$$

3. Boundary operators. If $u \in \mathscr{D}(\bar{\Omega})$ we define (using the notation of Lions and Magenes [16]) the map $\gamma_{0}$ by $\gamma_{0} u=u$ restricted to $\Gamma$, and set $\gamma_{k} u=\gamma_{0}(\partial / \partial \nu)^{k} u$ where $\nu$ denotes the normal to $\Gamma$.

LeMma (Ehrling [8]) 3.1. The map $u \rightarrow \gamma_{k} u$ is a continuous map of $\mathscr{D}(\bar{\Omega})$ with the topology induced by $H^{k+1}(\Omega)$ into $H^{\circ}(\Gamma)$ and thus has a continuous extension to $H^{k+1}(\Omega)$. If $\varepsilon>0$ there exists a $C(\varepsilon)>0$ such that for $u \in H^{k+1}(\Omega)\left\|\gamma_{k} u\right\|_{L^{2}(\Gamma)} \leqq \varepsilon\|u\|_{k+1}+C(\varepsilon)\|u\|_{0}$.

This is proved using a partition of unity just as in the usual proof for compact regions. A detailed proof appears in the thesis of 
Beals [4] so we do not include it here.

Since $\gamma_{0}$ is continuous, its kernel is a closed subspace of $H^{m}(\Omega)$ for $m$ a positive integer. Thus $H^{m}(\Omega) / \operatorname{ker} \gamma_{0}$ is a Hilbert space with the quotient topology.

Definition 3.2. The image of $H^{m}(\Omega)$ under the map $\gamma_{0}$ with the topology of $H^{m}(\Omega) / \operatorname{ker} \gamma_{0}$ is defined to be the space $H^{m-(1 / 2)}(\Gamma)$. The norm is written $\|\cdot\|_{m-(1 / 2), \Gamma}$. It follows that $\gamma_{0}$ is a topological homomorphism of $H^{m}(\Omega)$ onto $H^{m-(1 / 2)}(\Gamma)$.

If $\Omega$ is the half space $\left\{x \in R^{n}: x_{n}>0\right\}$ so that $\Gamma$ is $R^{n-1}$ and if $x^{\prime}$ denotes points in $R^{n-1}$ and $\xi^{\prime}$ points in the dual space, then

$$
H^{m-(1 / 2)}(\Gamma)=\left\{f \in L^{2}(\Gamma): \int\left(1+\left|\xi^{\prime}\right|^{2}\right)^{m-(1 / 2)}\left|\hat{f}\left(\xi^{\prime}\right)\right|^{2} d \xi^{\prime}<\infty\right\}
$$

and

$$
\|f\|_{m-(1 / 2), \Gamma}=\left(\int\left(1+\left|\xi^{\prime}\right|^{2}\right)^{m-(1 / 2)}\left|\hat{f}\left(\xi^{\prime}\right)\right|^{2} d \xi^{\prime}\right)^{1 / 2} .
$$

Using a partition of unity and local homeomorphisms just as when $\Gamma$ is compact another definition of $H^{m-(1 / 2)}(\Gamma)$ (and in fact $H^{s}(\Gamma)$ for $s$ real) can be given and the two definitions yield the same spaces. It is worth mentioning that difficulties appear when $p \neq 2$, see for example Lions-Magenes [17].

Definition 3.3. If $s$ is real and $m<s<m+1$ define $H^{s}(\Gamma)=$ $\left[H^{m}(\Gamma), H^{m+1}, \delta(s-m)\right]$.

THeOREM 3.4. For $s \geqq 0$ the space $H^{s}(\Gamma)$ coincides with the space defined in the discussion preceding Definition 3.3.

Lemma 3.5. $\mathscr{D}(\Gamma)$ is dense in $H^{s}(\Gamma)$.

LEMma 3.6. Given $\varepsilon>0$ there exists a $C(\varepsilon)>0$ such that for $0 \leqq s<t$ and $u \in \mathscr{D}(\Gamma),\left\|\gamma_{j} u\right\|_{s} \leqq \varepsilon\left\|\gamma_{j} u\right\|_{t}+C(\varepsilon)\left\|\gamma_{j} u\right\|_{0}$.

THEOREM 3.7. Let $s>1 / 2$ be real and $m=[s]$ the greatest integer $\leqq s$. The map $u \rightarrow \gamma u=\left(\gamma_{0} u, \cdots, \gamma_{m-1} u\right)$ is a continuous linear map of $\mathscr{D}(\bar{\Omega})$ with the topology induced by $H^{s}(\Omega)$ onto $\prod_{j=0}^{m-1} \mathscr{D}(\Gamma)$ with the topology induced by $\prod_{j=0}^{m-1} H^{s-j-(1 / 2)}(\Gamma) . \quad \gamma$ can be extended continuously to $H^{s}(\Omega)$ and yields a continuous linear map of $H^{s}(\Omega)$ onto $\prod_{j=0}^{m-1} H^{s-j-(1 / 2)}(\Gamma)$. The kernel of $\gamma$ is $H_{0}^{s}(\Omega)$.

Now let $B_{0}, \cdots, B_{r-1}$ be a family of $r$ differential operators with infinitely differentiable coefficients defined on some open set $\Omega^{\prime}$ containing $\bar{\Omega}$. 
Definition [2] 3.8. The set $B_{0}, B_{1}, \cdots, B_{r-1}$ is called a Dirichlet set of order $r$ if and only if

(1) $B_{k}=b_{k}(x) \gamma_{k}+\Lambda_{k}$ where the order of $B_{k}$ is $k$ and the normal order of $\Lambda_{k}$ is $\leqq k-1$.

(2) $\inf \left\{\left|b_{k}(x)\right|: 0 \leqq k \leqq r-1, x \in \Gamma\right\}>0$.

A subset of a Dirichlet set the highest order appearing being $p$ is called a normal set of order $p$. A set of differential operators having the properties of a normal set can be extended in many ways to form a Dirichlet set.

TheOREM 3.9. Let $B=\left\{B_{0}, \cdots, B_{m-1}\right\}$ be a normal set of order $M_{0}$, the order of $B_{j}$ being $m_{j}$. If $s$ is a real number, $s>M_{0}+1 / 2$, then the map $u \rightarrow B u=\left(B_{0} u, \cdots, B_{m-1} u\right)$ is a continuous linear map of $\mathscr{D}(\bar{\Omega})$ with the topology induced by $H^{s}(\Omega)$ onto $\prod_{j=0}^{m-1} \mathscr{D}(\Gamma)$ with the topology induced by $\Pi_{j=0}^{m-1} H^{s-m_{j}-(1 / 2)}(\Gamma)$ and can be extended continuously so as to be a homomorphism of $H^{s}(\Omega)$ onto $\prod_{\jmath=0}^{m-1} H^{s-m_{j}-\{1 / 2)}(\Gamma)$. If $B$ is a Dirichlet set, the kernel of $B$ is $H_{0}^{s}(\Omega)$.

4. Closable elliptic operators. Let $A=\sum_{|\alpha|,|\beta| \leqq m} D^{\beta} a_{\alpha \beta}(x) D^{\alpha}$ be a differential operator of order $2 m$ with infinitely differentiable coefficients defined on an open set $\Omega^{\prime}$ containing $\bar{\Omega}$. Let

$$
A(x, \xi)=\sum_{|\alpha|=|\beta|=m} a_{\alpha \beta}(x) \xi^{\alpha+\beta} \text {. }
$$

Definition 4.1. (i) $A$ is uniformly elliptic if and only if there exists a number $a_{0}>0$ such that for $x \in \bar{\Omega},\left|A_{0}(x, \xi)\right| \geqq a_{0}|\xi|^{2 m}$.

(ii) $A$ is properly elliptic if and only if for each $x \in \Gamma$ denoting by $\nu_{x}$ the inner directed unit normal to $\Gamma$ at $x$ and $\tau_{x}$ a unit tangent vector to $\Gamma$ at $x$ the polynomial in $\lambda A_{0}\left(x, \tau_{x}+\lambda \nu_{x}\right)$ has precisely $m$ roots with positive imaginary part and $m$ roots with negative imaginary part. It is known that when $n>2$ every elliptic operator is properly elliptic.

We now introduce a condition on boundary operators associated with $A$ which has almost as many names as there are authors who have introduced it. It has been called the complementing condition by Agmon, Douglis and Nirenberg [1], regularity condition by Browder [5], and the boundary operators are said to cover $A$ by Schechter [23].

Definition 4.2. Let $B=\left(B_{0}, \cdots, B_{m-1}\right)$ be a normal ${ }^{1}$ set of boundary operators, the order of $B_{j}$ being $m_{j}$ and $m_{j}<2 m$. Let $B_{j}=$ $b_{j}(x) \gamma_{m_{j}}+\Lambda_{j}$ and $C(x, \tau)$ be a closed Jordan curve in the upper half plane containing those roots of $A_{0}\left(x, \tau_{x}+\lambda \nu_{x}\right)$ with positive imaginary part. Set

${ }^{1}$ Normality is not essential for this definition. 


$$
p_{j k}(x)=\int_{0} \lambda^{k-1} b_{j}\left(x, \tau_{x}+\lambda \nu_{x}\right)\left[A_{0}\left(x, \tau_{x}+\lambda \nu_{x}\right)\right]^{-1} d \lambda .
$$

The system $(A, B)$ will be called closable, elliptic if and only if $\inf \left\{\left|p_{j k}(x)\right| \geqq a>0, x \in \Gamma,\left|\tau_{x}\right|=1,0 \leqq j \leqq m-1,1 \leqq k \leqq m\right\}$. We are using the form as given in Browder [5].

Notation 4.3. We will denote the formal adjoint of $A$ by $A^{\prime}$.

If $B$ is a given normal set of orders $\leqq 2 m-1, B=\left(B_{0}, \cdots, B_{m-1}\right)$, let $C=\left(C_{0}, \cdots, C_{m-1}\right)$ be another normal set such that the set $(B, C)$ is a Dirichlet set of order $2 m$. We let $m_{j}$ be the order of $B_{j}$ and $\mu_{j}$ be the order of $C_{j}, j=0, \cdots, m-1$. The following result is basic. We assume all operators have infinitely differentiable coefficients.

THEOREM 4.4. Given an elliptic differential operator of order $2 m$ and a Dirichlet set $(B, C)$ of order $2 m$, there exists another Dirichlet set $\left(B^{\prime}, C^{\prime}\right)$ having infinitely differentiable coefficients with the order of $B_{j}^{\prime}=m_{j}^{\prime}=2 m-1-\mu_{j}$, and the order of $C_{j}^{\prime}=\mu_{j}^{\prime}=$ $2 m-1-m_{j}, j=0, \cdots, m-1$, and such that for $u, v \in \mathscr{D}(\bar{\Omega})$

$$
(A u, v)-\left(u, A^{\prime} v\right)=\sum_{j=0}^{m-1}\left[\int_{T^{\prime}} C_{j} u \overline{B_{j}^{\prime} v} d \sigma-\int_{\Gamma} B_{j} u \overline{C_{j}^{\prime} v} d \sigma\right] .
$$

If $(A, B)$ is closable elliptic then $\left(A^{\prime}, B^{\prime}\right)$ is also closable elliptic.

We will refer to (4.1) as Green's formula. A proof can be found in Schechter [24].

Assumption $I$ 4.5. We will always assume that $(A, B)$ is closable elliptic unless something is stated to the contrary.

We will need the following

Definition (Lions-Magenes [16]) 4.6. For $0 \leqq s$ let

$$
\mathscr{D}_{A}^{s}(\Omega)=\left\{u \in H^{s}(\Omega): A u \in L^{2}(\Omega)\right\} .
$$

Equip $\mathscr{D}_{A}^{s}(\Omega)$ with the norm $\|u\|_{s, A}=\left(\|u\|_{s}^{2}+\|A u\|_{0}^{2}\right)^{1 / 2}$ which makes it a Hilbert space.

Proposition 4.7. Let $V, H$ be Hilbert spaces with $V \subset H$. Let $T \in \mathscr{L}(V, H)$; then the graph of $T$ is closed in $H \times H$ if and only if there exists a constant $K$ such that for $u \in V,\|u\|_{V} \leqq K\left[\|T u\|_{H}+\right.$ $\left.\|u\|_{H}\right]$.

Lemma 4.8. The map $D_{j} \in \mathscr{S}\left(H^{s}(\Omega), H^{s-1}(\Omega)\right)$ for $s$ real, $s \neq 1 / 2$. 


\section{Proof. Lions-Magenes $[17,18]$.}

DeFINITION 4.9. Let $V_{B}(\Omega)=\{u \in \mathscr{D}(\bar{\Omega}): B u=0\}$.

THEOREM 4.10. There exists a constant $C_{2 m}>0$ such that for $u \in V_{B}(\Omega),\|u\|_{2 m} \leqq C_{2 m}\left[\|A u\|_{0}+\|u\|_{0}\right]$.

Proof. This inequality has been proved by a great many authors, for example Browder [5], Agmon, Douglis and Nirenberg [1], Schechter [23].

REMARK 4.11. It follows that for $u \in V_{B^{\prime}}(\Omega)$, $\|u\|_{2 m} \leqq C_{2 m}\left[\left\|A^{\prime} u\right\|_{0}+\right.$ $\left.\|u\|_{0}\right]$ for some $C_{2 m}>0$.

Definition 4.12. The closure of $V_{B}$ in the topology of $H^{s}(\Omega)$ will be denoted by $V_{B}^{s}(\Omega)$.

CoRollary 4.13. The operator $A\left(A^{\prime}\right)$ with domain $V_{B}^{2 m}(\Omega)\left(V_{B^{\prime}}^{2 m}(\Omega)\right)$ is a closed operator in $L^{2}(\Omega)$.

REMARK 4.14. There are numerous generalizations of Theorem 4.10 now in the literature. Some of these will be discussed in what follows.

Theorem (Browder [5], Schechter [24]) 4.15. Let $A_{B}$ be the operator $A$ with domain $V_{B}^{2 m}(\Omega)$ and $A_{B^{\prime}}^{\prime}$ the operator $A^{\prime}$ with domain $V_{B^{\prime}}^{2 m}(\Omega)$. Regarding each as an operator in $L^{2}(\Omega)$ the adjoint of $A_{B}$. is $A_{B^{\prime}}^{\prime}$.

THEOREM 4.16. There exists a constant $C_{2 m}$ such that for $u \in \mathscr{D}(\bar{\Omega})$

$$
\|u\|_{2 m} \leqq C_{2 m}\left[\|A u\|_{0}+\|u\|_{0}+\sum_{j=0}^{m-1}\left\|B_{j} u\right\|_{2 m-m_{j}-(1 / 2)}\right] .
$$

Proof. Theorem 4.10, Lemma 4.8 and Theorem 3.9. This result appears for example in Agmon, Douglis and Nirenberg [1].

COROLlaRy 4.17. If $\Omega$ is relatively compact then the kernel of $A_{B}\left(A_{B^{\prime}}^{\prime}\right)$ is finite dimensional, the range is closed and has finite co-dimension.

Proof. This follows by Rellich's lemma, i.e., the cannonical injection of $H^{k}(\Omega)$ into $H^{j}(\Omega)(\mathrm{k}>j)$ is compact; see Browder [5]. 
5. Some interpolation results and normally solvable operators.

Definition 5.1. The Dirichlet operator is the operator $A$ with domain $V_{r}^{2 m}(\Omega)$.

THEOREM (Lions and Magenes [19]) 5.2. If the Dirichlet operator is an isomorphism and $H_{0}=\mathscr{D}_{A}^{0}(\Omega), H_{1}=\mathscr{D}_{A}^{2 m}(\Omega)$, then for $0 \leqq \theta \leqq 1$, $H_{\theta}=\mathscr{D}_{A}^{2 m \theta}(\Omega)$, so long as $2 m \theta \neq s+(1 / 2)$, s an integer $\geqq 0$.

Proof. This follows from Propositions 5.4 and 5.6 of Lions-Magenes $\mathrm{V}$ [19], Theorem 2.8 and the fact that for $s=0$ and $2 m$ the map $(A, \gamma)$ is an isomorphism of $\mathscr{D}_{A}^{s}(\Omega)$ onto $H^{0}(\Omega) \times \prod_{j=0}^{m-1} H^{s-j-(1 / 2)}(\Gamma)$ and of course Theorem 2.6.

Definition 5.3. For $0 \leqq s \leqq 2 m$, let $\tilde{\mathscr{D}}_{A}^{s}(\Omega)$ be the spaces interpolated between $\mathscr{D}_{A}^{0}(\Omega)$ and $\mathscr{D}_{A}^{2 m}(\Omega)$.

Thus if the Dirichlet operator is an isomorphism, Theorem 5.2 says that $\tilde{\mathscr{D}}_{A}^{s}(\Omega)$ can be identified with $\mathscr{D}_{A}^{s}(\Omega)$ for $0 \leqq s \leqq m$ so long as $s-(1 / 2)$ is not an integer. Observe that $\tilde{\mathscr{D}}_{A}^{s}(\Omega) \subset \mathscr{D}_{A}^{s}(\Omega)$ always the injection being continuous. We shall identify the spaces $\tilde{\mathscr{D}}_{A}^{s}(\Omega)$ in what follows.

Theorem 5.4. If $(B, C)$ is a Dirichlet set of order $2 m$, the map $(B, C)$ is a continuous linear map of $\tilde{\mathscr{D}}_{A}^{s}(\Omega)$ into $\prod_{j=0}^{m-1} H^{s-m_{j-(1 / 2)}}(\Gamma) \times$ $\prod_{j=0}^{m-1} H^{s-\mu_{j}-(1 / 2)}$ for $0 \leqq s \leqq 2 m$.

Proof. For $s=2 m$ this is just Theorem 3.9 and for $s=0$ it is proved in Lions-Magenes [16]. For $0<s<2 m$, use interpolation.

THEOREM 5.5. For $u \in \mathscr{D}_{A}^{s}(\Omega)$ and $v \in \mathscr{D}_{A^{\prime}}^{2 m-s}(\Omega)$,

$$
(A u, v)-\left(u, A^{\prime} v\right)=\sum_{j=0}^{m-1}\left\{\left\langle C_{j} u, \overline{B_{j}^{\prime} v}\right\rangle-\left\langle B_{j} u, \overline{C_{j}^{\prime} v}\right\rangle\right\},
$$

then brackets representing the duality between $\prod_{j=0}^{m-1} H^{s-\mu_{j}-(1 / 2)}(\Gamma)$ and $\prod_{j=0}^{m-1} H^{2 m-s-m_{j}^{\prime}-(1 / 2)}(\Gamma)$ and $\prod_{j=0}^{m-1} H^{s-m_{j}-(1 / 2)}(\Gamma)$ and $\prod_{j=0}^{m-1} H^{2 m-s-\mu_{j}^{\prime}-(1 / 2)}(\Gamma)$.

Definition 5.6. Let $N_{B}\left(N_{B^{\prime}}\right)$ be the kernel of $A_{B}\left(A_{B^{\prime}}^{\prime}\right)$.

THEOREM 5.7. For $0 \leqq s \leqq 2 m$ let $N_{s}$ denote $N_{B}$ equipped with the topology of $H^{s}(\Omega)$ and $N_{-s}$ denote $N_{B}$ equipped with the topology $H_{\bar{L}}^{-s}$. For $-2 m \leqq s, t \leqq 2 m \quad N_{s}$ is topologically isomorphic to $N_{t}$ 
and is thus closed in each such topology.

Proof. Freeman [10].

Definition 5.8. For $0 \leqq s \leqq 2 m$ let $N_{s, B}^{\llcorner}=\left\{u \in H^{s}(\Omega):(u, v)_{0}=0\right.$ for $\left.v \in N_{B}\right\}$ and $N_{-s, B}^{\perp}=\left\{u \in H_{\bar{\Omega}}^{-t}:(u, v)_{0}=0\right.$ for $\left.v \in N_{B}\right\}$.

LEMma 5.9. Let $P$ be the orthogonal projection of $H^{0}$ onto $N_{B}^{0}$ and $0 \leqq s \leqq 2 m$. Then $P \in \mathscr{S}\left(H^{s}(\Omega), H^{s}(\Omega)\right)$ and $P \in \mathscr{S}\left(H_{\bar{\Omega}}^{-s}, H_{\bar{\Omega}}^{-s}\right)$.

THEOREM 5.10. Let $0 \leqq s \leqq 2 m$; then each $u \in H^{s}(\Omega)\left(H_{\overline{\bar{a}}}^{-s}\right)$ can be written uniquely in the form $u=u^{\prime}+u^{\prime \prime}$ with $u^{\prime} \in N_{B}$ and $u^{\prime \prime} \in N_{s, B}^{\llcorner}\left(N_{-s, B}^{\perp}\right)$.

Proofs can be found in Freeman [10]. Corresponding results hold for $N_{B^{\prime}}, N_{B^{\prime}, s}^{\perp}$.

THEOREM 5.11. Let $V$ and $H$ be Hilbert spaces with $V \subset H$. Let $T \in \mathscr{S}(V, H)$ and assume $T$ is a closed linear operator in $H$. Let $N$ denote the kernel of $T$ and $N_{\dot{V}}=\left\{u \in V:(u, v)_{H}=0\right.$ for $\left.v \in N\right\}$. Then the range of $T$ is closed in $H$ if and only if there exists a constant $C>0$ such that for $u \in N_{V}^{\llcorner},\|u\|_{\nabla} \leqq C\|T u\|_{H}$.

Proof. Open mapping theorem.

Theorem (Kato [11]) 5.12. Let $T$ be a closed densely defined linear operator mapping a Banach space $E$ into a Banach space $F . T^{\prime}$ the adjoint of $T$. Then the range of $T$ is closed in $F$ if and only if the range of $T^{\prime}$ is closed in $E^{\prime}$.

Assumption 5.13. In the remainder of this section we assume that the operator $A_{B}$ (and thus also $A_{B^{\prime}}^{\prime}$ ) has closed range in $H^{\circ}(\Omega)$.

Lemma 5.14. Let $f \in H^{\circ}(\Omega)$ and

$$
\varphi=\left(\varphi_{0}, \cdots, \varphi_{m-1}\right) \in \prod_{j=0}^{m-1} H^{2 m-m_{j}-(1 / 2)}(\Gamma) .
$$

There exists a $u \in H^{2 m}(\Omega)$ such that $A u=f$ and $B u=\varphi$ if and only if for all $v \in N_{B^{\prime}}$ :

$$
(f, v)+\sum_{j=0}^{m-1}\left\langle\varphi_{j}, \overline{C_{j}^{\prime} v}\right\rangle=0 .
$$

Proof. If such a $u$ exists then by Green's formula the condition is clearly verified. 
Now suppose $f$ and $\varphi$ are given subject to the orthogonality condition (5.1). There exists $u_{1} \in H^{2 m}(\Omega)$ such that $B u_{1}=\varphi$. Let $A u_{1}=f_{1}$ and apply Green's formula to $u_{1}$ and $v \in H^{2 m}(\Omega)$ to obtain

$$
\left(f_{1}, v\right)+\sum_{j=0}^{m-1}\left\langle\varphi_{j}, \overline{C_{j}^{\prime} v}\right\rangle=\left(u_{1}, A^{\prime} v\right)-\sum_{j=0}^{m-1}\left\langle C_{j} u_{1}, \overline{B_{j}^{\prime} v}\right\rangle .
$$

Thus for $v \in N_{B^{\prime}}$ we have $\left(f_{1}, v\right)+\sum_{i j=0}^{m-1}\left\langle\varphi_{j}, \overline{C_{j}^{\prime} v}\right\rangle=0$ and using the condition of the theorem we have $\left(f-f_{1}, v\right)=0$ for all $v \in N_{B^{\prime}}$. But by Assumption 5.13 there exists $u_{2} \in V_{B}^{2 m}(\Omega)$ such that $A u_{2}=f-f_{1}$. Then $u=u_{1}+u_{2} \in H^{2 m}(\Omega)$ and $A u=f, B u=\varphi$.

The set of $f, \varphi, f \in H^{0}(\Omega), \varphi \in \prod_{j=0}^{m-1} H^{2 m-m_{j}-(1 / 2)}(\Gamma)$ which satisfy (5.1) is clearly closed.

Corollary 5.15. ( i ) ( $A, B)$ maps $H^{2 m}(\Omega)$ onto the closed subspace

$$
\begin{gathered}
\left\{(f, \varphi): f \in H^{2 m}(\Omega), \varphi \in \sum_{j=0}^{m-1} H^{2 m-m_{j}-(1 / 2)}(\Gamma):(f, v)\right. \\
+\sum_{j=0}^{m-1}\left\langle\varphi_{j}, \overline{\left.C_{j}^{\prime} v\right\rangle}=0, v \in N_{B^{\prime}}\right\} .
\end{gathered}
$$

(ii) $(A, B)$ is an isomorphism of $N_{2 m, B}^{\perp}$ onto its range and for

$$
u \in N_{2 m, B}^{\perp},\|u\|_{2 m} \leqq C_{2 m}^{\prime}\left[\|A u\|_{0}+\sum_{j=0}^{m-1}\left\|B_{j} u\right\|_{2 m-m_{j}-(1 / 2)}\right] .
$$

Lemma 5.16. For $f \in H^{\circ}(\Omega)$ and $\varphi \in \prod_{j=0}^{m-1} H^{-m_{j-(1 / 2)}}(\Gamma)$ there exists a $u \in \mathscr{D}_{A}^{0}(\Omega)$ such that $A u=f$ and $B u=\varphi$ if and only if there exists a constant $K$ such that for all $v \in V_{B^{\prime}}^{2 m}(\Omega)$

$$
\left|(f, v)_{0}+\sum_{j=0}^{m-1}\left\langle\varphi_{j}, \overline{C_{j}^{\prime} v}\right\rangle\right| \leqq K\left\|A^{\prime} v\right\|_{0} .
$$

Proof. Suppose such a $u$ exists and $v \in V_{B^{\prime}}^{2 m}(\Omega)$. Then

$$
(f, v)_{0}+\sum_{j=0}^{m-1}\left\langle\varphi_{j}, \overline{C_{j}^{\prime} v}\right\rangle=\left(u, A^{\prime} v\right)
$$

and (5.2) is satisfied with $\|u\|_{0}=K$. Now suppose (5.2) is satisfied and define $F\left(A^{\prime} v\right)=(f, v)+\sum_{j=0}^{m-1}\left\langle\varphi_{j}, C_{j}^{\prime} v\right\rangle$. By (5.2), $F$ is a continuous conjugate linear form on a closed subspace of $H^{0}(\Omega)$ and has a continuous extension to all of $H^{0}(\Omega)$. Thus there exists $u \in H^{0}(\Omega)$ such that $\left(u, A^{\prime} v\right)=(f, v)+\sum_{j=0}^{m-1}\left\langle\varphi_{j}, \overline{C_{\jmath}^{\prime} v}\right\rangle$ for $v \in V_{B^{\prime}}^{2 m}(\Omega)$. Taking $v \in \mathscr{D}(\Omega)$ we see that $u \in \mathscr{D}_{A}^{0}(\Omega)$ and $A u=f$. But then $\left(u, A^{\prime} v\right)=(A u, v)+$ $\sum_{j=0}^{m-1}\left\langle B_{j} u, \overline{C_{j}^{\prime} v}\right\rangle$ and since it is clear from Theorem 3.9 that $C^{\prime} v$ fills up $\prod_{j=0}^{m-1} H^{2 m-\mu_{j}^{\prime}-(1 / 2)}(\Gamma)$ as $v$ runs through $V_{B^{\prime}}^{2 m}$ it follows that $B u=\varnothing$.

Theorem 5.17. Let $f \in H^{0}(\Omega)$ and $\varphi \in \prod_{j=0}^{m-1} H^{-m_{j}-(1 / 2)}(\Gamma)$. There 
exists $u \in \mathscr{D}_{A}^{0}(\Omega)$ such that $A u=f$ and $B u=\varnothing$ if and only if for all $v \in N_{B^{\prime}}$

$$
(f, v)+\sum_{j=0}^{m-1}\left\langle\varphi_{j}, \overline{C_{\jmath}^{\prime} v}\right\rangle=0
$$

Proof. Let $v \in V_{B^{\prime}}^{2 m}(\Omega)$ and write $v=v^{\prime}+v^{\prime \prime}$ with $v^{\prime} \in N_{B^{\prime}}$ and $v^{\prime \prime} \in N_{B^{\prime}, 2 m}^{\prime}$. Since $N_{B^{\prime}} \subset V_{B^{\prime}}^{2 m}$ it follows that $v^{\prime \prime} \in V_{B^{\prime}}^{2 m}$ and by Assumption 5.13 and Theorem 5.11 there exists a constant $K$ such that $\left\|v^{\prime \prime}\right\|_{2 m} \leqq$ $K\left\|A^{\prime} v\right\|_{0}$. Now using (5.3) we have for

$$
v \in V_{B^{\prime}}^{2 m}:(f, v)+\sum_{j=0}^{m-1}\left\langle\varphi_{j}, \overline{C_{\jmath}^{\prime} v}\right\rangle=\left(f, v^{\prime \prime}\right)+\sum_{j=0}^{m-1}\left\langle\varphi_{j}, \overline{C_{j}^{\prime} v^{\prime \prime}}\right\rangle
$$

and thus

$$
\begin{aligned}
\mid(f, v)+\sum_{j=0}^{m-1}\left\langle\varphi_{j}, \overline{\left.C_{j}^{\prime} v\right\rangle \mid}\right. & \leqq\|f\|_{-2 m}\left\|v^{\prime \prime}\right\|_{2 m}+K^{\prime} \sum_{j=0}^{m-1}\left\|\varphi_{j}\right\|_{-m_{j}-(1 / 2)}\left\|v^{\prime \prime}\right\|_{2 m} \\
& \leqq K\left(\|f\|_{-2 m}+K^{\prime} \sum_{j=0}^{m-1}\left\|\varphi_{j}\right\|_{-m_{j}-(1 / 2)}\right)\left\|A^{\prime} v\right\|_{0}
\end{aligned}
$$

Now apply Lemma 5.16 .

Corollary 5.18. $(A, B)$ maps $\mathscr{D}_{A}^{0}(\Omega)$ onto the closed subspace of $H^{0}(\Omega) \times \prod_{j=0}^{m-1} H^{-m_{j}-(1 / 2)}(\Gamma)$ consisting of those $(f, \varphi)^{\prime} s$ which satisfy $(5.3)$

The kernel of $A, B$ in $\mathscr{D}_{A}^{0}(\Omega)$ is just $N_{B}$ and $N_{B, 0}^{\perp} \cap \mathscr{D}_{A}^{0}(\Omega)$ is closed in $\mathscr{D}_{A}^{0}(\Omega)$.

Corollary 5.19. $(A, B)$ is an isomorphism of $N_{B, 0}^{\perp} \cap \mathscr{D}_{A}^{0}(\Omega)$ onto its range. There exists a constant $C_{0}^{\prime}>0$ such that $\|u\|_{0} \leqq$ $C_{0}^{\prime}\left[\|A u\|_{0}+\sum_{j=0}^{m-1}\left\|B_{j} u\right\|_{-m_{j}-(1 / 2)}\right]$ for $u \in N_{B, 0}^{\lrcorner} \cap \mathscr{D}_{A}^{0}(\Omega)$.

Now by Corollary $5.15(A, B)$ maps $H^{2 m}(\Omega)$ onto a closed subspace of $H^{0}(\Omega) \times \Pi_{j=0}^{m-1} H^{2 m-m_{j}-(1 / 2)}(\Gamma)$ and by Corollary $5.18(A, B)$ maps $\mathscr{D}_{A}^{0}(\Omega)$ onto a closed subspace of $H^{0}(\Omega) \times \prod_{j=0}^{m-1} H^{-m_{j}-(1 / 2)}(\Gamma)$. Thus to each element $\left(f, \varphi_{0}, \cdots, \varphi_{m-1}\right)$ in the image of $(A, B)$ in $H^{0}(\Omega) \times$ $\prod_{j=0}^{m-1} H^{\left.s-m_{j-1}-1 / 2\right)}(\Gamma), s=0,2 m$, there corresponds a unique $u \in \mathscr{D}_{A}^{s}(\Omega) \cap N_{\bar{B}, s}^{\perp}$ for $s=0,2 m$, such that $A u=f$ and $B u=\varphi$. Define a map $T$ by $T(f, \varphi)=u$. By Corollary 5.15 and Corollary $5.19 T$ is a continuous map of the image of $(A, B)$ into $\mathscr{D}_{A}^{0}(\Omega)$ and into $\mathscr{D}_{A}^{2 m}(\Omega)$. By letting $T$ be zero in the orthogonal complement of the image of $(A, B)$ in

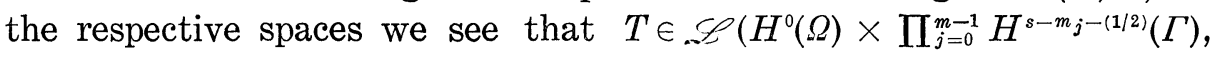
$\mathscr{D}_{A}^{s}(\Omega)$ ) for $s=0,2 m$ and thus by interpolation for $0<s<2 m$, using a result of Lions [14]. 
Theorem 5.20. (i ) For $u \in \tilde{\mathscr{D}}_{A}^{s}(\Omega) \cap N_{s_{1} B}^{\perp}, 0 \leqq s \leqq 2 m$, there exists a constant $C_{s}^{\prime}$ such that

$$
\|u\|_{s} \leqq C_{s}^{\prime}\left[\|A u\|_{0}+\sum_{j=0}^{m-1}\left\|B_{j} u\right\|_{s-m_{j}-(1 / 2)}\right] .
$$

(ii) $(A, B)$ maps $\tilde{\mathscr{D}}_{A}^{s}(\Omega) \cap N_{s_{1} B}^{\perp}$ onto the closed subset of

$$
H^{0}(\Omega) \times \prod_{j=0}^{m-1} H^{s-m_{j}-(1 / 2)}(\Gamma)
$$

consisting of those $(f, \varphi)^{\prime}$ s in

such that

$$
H^{0}(\Omega) \times \prod_{\jmath=0}^{m-1} H^{s-m_{j}-(1 / 2)}(\Gamma)
$$

$$
(f, v)_{0}+\sum_{j=0}^{m-1}\left\langle\varphi_{j}, \overline{\left.C_{j}^{\prime} v\right\rangle}=0 \quad \text { for all } \quad v \in N_{B^{\prime}}^{0} .\right.
$$

(iii) There exists a constant $C_{s}$ such that for $u \in \tilde{\mathscr{D}}_{A}^{s}(\Omega)$

$$
\|u\|_{s} \leqq C_{s}^{\prime}\left[\|A u\|_{0}+\|u\|_{0}+\sum_{j=0}^{m-1}\left\|B_{j} u\right\|_{s-m_{j-(1 / 2)}}\right] .
$$

Proof. (i) Follows from the preceding discussion. This is essentially the argument used in a slightly different context in Schechter [25].

(ii) (5.4) implies that the image of $(A, B)$ is closed whereas the set of $(f, \varphi)$ satisfying (5.5) is clearly closed and contains the image of $(A, B)$. If the sets were not the same there would exist an

$$
(f, \varphi) \in H^{0}(\Omega) \times \sum_{j=0}^{m-1} H^{s-m_{j}-(1 / 2)}(\Gamma)
$$

satisfying (5.5) and a

$$
(v, \dot{\psi}) \in H^{0}(\Omega) \times \sum_{j=0}^{m-1} H^{2 m-s-u_{j}^{\prime}-(1 / 2)}(\Gamma)
$$

such that $(f, v)+\sum_{j=0}^{m-1}\left\langle\phi_{j}, \bar{\psi}_{j}\right\rangle \neq 0$ whereas $(A u, v)-\sum_{j=0}^{m-1}\left\langle B_{j} u, \bar{\psi}_{j}\right\rangle=0$ for $u \in \mathscr{D}_{A}^{s}(\Omega)$. It follows in particular that $(A u, v)=0$ for $u \in V_{B}$ and thus $v \in N^{\prime}$. Hence $(A u, v)-\sum_{j=0}^{m-1}\left\langle B_{j} u, \overline{C_{j}^{\prime} v}\right\rangle=0$ for $u \in \tilde{\mathscr{D}}_{A}^{s}(\Omega)$. It follows then that $C_{j}^{\prime} v=\psi_{j}$ and thus that $(f, v)+\sum_{j=0}^{m-1}\left\langle\varphi_{j}, \overline{\left.C_{j}^{\prime} v\right\rangle \neq 0}\right.$ for $v \in N_{B^{\prime}}^{0}$ which contradicts (5.4).

(iii) If $u \in \tilde{\mathscr{V}}_{A}^{s}(\Omega)$, with $u=u^{\prime}+u^{\prime \prime}$ with $u^{\prime} \in N$ and

$$
u^{\prime \prime} \in N_{B, s}^{\perp} \cap \tilde{\mathscr{D}}_{A}^{s}(\Omega),
$$

we have 


$$
\begin{aligned}
\|u\|_{s} \leqq\left\|u^{\prime}\right\|_{s} & +\left\|u^{\prime \prime}\right\|_{s} \leqq\left\|u^{\prime}\right\|_{s} \\
& +C_{s}^{\prime}\left[\|A u\|_{0}+\sum_{\jmath=0}^{m-1}\left\|B_{\jmath} u\right\|_{s-m_{j}-(1 / 2)}+\sum_{j=0}^{m-1}\left\|B_{j} u^{\prime}\right\|_{s-m_{j}-(1 / 2)}\right]
\end{aligned}
$$

since $A u^{\prime}=0$. But $B_{j}$ is continuous on $\mathscr{D}_{A}^{s}(\Omega)$ and $A u^{\prime}=0$ so $\left\|B_{j} u^{\prime}\right\|_{s-m_{j}-(1 / 2)} K\left\|u^{\prime}\right\|_{s}$. But using Theorem 5.7, $\left\|u^{\prime}\right\|_{s} \leqq K\left\|u^{\prime}\right\|_{0} \leqq K\|u\|_{0}$ by Lemma 5.9 .

When $s-(1 / 2)$ is not an integer, $\tilde{\mathscr{D}}_{A}^{s}(\Omega)$ can be identified. In general assuming 5.13, the closed range assumption, as was remarked in [10], using the results of [10] and an argument similar to that which preceded Theorem 5.20 one can show that (5.4) and (5.6) are true for $u \in \mathscr{D}(\bar{\Omega}) \cap N_{\bar{B} s}^{\perp}$ and $u \in \mathscr{D}(\bar{\Omega})$ respectively.

DeFinition 5.21. For $u \in \mathscr{D}(\bar{\Omega})$ let

$$
\left\|\left.u\right|_{\hat{\mathscr{E}}_{A}^{s}(\Omega)} ^{2}=\right\| u\left\|_{s}^{2}+\right\| A u\left\|_{0}^{2}+\sum_{j=0}^{m}\right\| B_{j} u \|_{s-m_{j}-(1 / 2)}^{2}
$$

and let $\hat{\mathscr{D}}_{A}^{s}(\Omega)$ be the completion of $\mathscr{D}(\bar{\Omega})$ in this norm.

By the preceding remarks $(A, B)$ maps $\hat{\mathscr{D}}_{A}^{s}(\Omega)$ onto a closed subspace of $H^{0}(\Omega) \times \prod_{j=0}^{m-1} H^{s-m_{j}-(1 / 2)}(\Gamma)$. Now $\hat{\mathscr{D}}_{A}^{s}(\Omega)$ is continuously embedded in $\mathscr{D}_{A}^{s}(\Omega), \mathscr{D}(\bar{\Omega})$ is dense in $\tilde{\mathscr{D}}_{4}^{s}(\Omega)$ and each $B_{j}$ is continuous on $\tilde{\mathscr{D}}_{A}^{s}(\Omega)$. It follows that $\tilde{\mathscr{D}}_{A}^{s}(\Omega)$ is continuously embedded in $\hat{\mathscr{D}}_{A}^{s}(\Omega)$. Since on the one hand the image under $(A, B)$ of $\tilde{\mathscr{D}}_{A}^{s}(\Omega)$ is characterized by (5.4) while on the other hand the image under $(A, B)$ of $\hat{\mathscr{D}}_{A}^{s}(\Omega)$ satisfies that condition, the two images must be the same. Thus if $u \in \hat{\mathscr{D}}_{A}^{s}(\Omega)$ there exists $v \in \tilde{\mathscr{D}}_{A}^{s}(\Omega)$ such that $A(u-v)=0$ and $B_{j}(u-v)=0$. Consequently $u-v \in N \subset \tilde{\mathscr{D}}_{A}^{s}(\Omega)$. Thus

Theorem 5.22. Under assumption (5.13) and for $0 \leqq s \leqq 2 m$ the spaces $\hat{\mathscr{D}}_{A}^{s}(\Omega)$ and $\tilde{\mathscr{D}}_{A}^{s}(\Omega)$ are the same.

Following Schechter [25] for $u \in \mathscr{D}(\bar{\Omega})$ we define

$$
|u|_{-s}=\sup \left\{\left|(u, v)_{0}\right|: \text { for } v \in V_{B^{\prime}} \text { and }\|v\|_{s}=1\right\}
$$

where $s \geqq 0$. Observe that for $u \in \mathscr{D}(\bar{\Omega}) \quad|u|_{-s} \leqq\|u\|_{-s} \leqq\|u\|_{0}$. The following result was proved by Schechter [26]; see also Peetre [22].

THEOREM 5.23. Let $s \geqq 0$. There exists a constant $C_{-s}>0$ such that for $u \in \mathscr{D}(\bar{\Omega})$

$$
\|u\|_{-s} \leqq K\left(|A u|_{-2 m-s}+\sum_{\jmath=0}^{m-1}\left\|B_{\jmath} u\right\|_{-m_{j-s-(1 / 2)}}+\|u\|_{-2 m-s}\right) .
$$


Moreover, there exists a constant $C_{-s}^{\prime}>0$ such that for $u:(u, v)_{0}=0$, for all $v \in N$, then

$$
\|u\|_{-s} \leqq C_{-s}^{\prime}\left(|A u|_{-2 m-s}+\sum_{j=0}^{m-1}\left\|B_{j} u\right\|_{-m_{j-s-(1 / 2)}}\right) .
$$

REMARK 5.24. (i) Both Peetre and Schechter assume that $\Omega$ we relatively compact. However, as was remarked in [10], Schechter's proof can be adopted to situations where $\Omega$ is not relatively compact if $A_{B}$ has closed range.

(ii) Each result in this section has a corresponding analog if $A$ is replaced by $A^{\prime}$ and $B$ is replaced by $B^{\prime}$.

6. Perturbed boundary operators. Let $L=\left(L_{0}, \cdots, L_{m-1}\right)$ where $L_{j} \in \mathscr{P}\left(H^{2 m}(\Omega), H^{2 m-m_{j}-(1 / 2)}(\Gamma)\right)$ and

$$
\begin{aligned}
L_{j}=\sum_{k=0}^{m-1} L_{k j} C_{k}, \quad L_{k j} \in \mathscr{L}\left(H^{2 m-\mu_{k}-(1 / 2)}(\Gamma), H^{2 m-m_{j}-(1 / 2)}(\Gamma)\right), \\
k=0, \cdots, m-1 ; \\
j=0, \cdots, m-1 .
\end{aligned}
$$

Moreover, let $L^{\prime}=\left(L_{0}^{\prime}, \cdots, L_{m-1}^{\prime}\right)$ where $L_{j}^{\prime}=\sum_{k=0}^{m-1} L_{j k}^{\prime} C_{k}^{\prime}$ and

$$
\begin{gathered}
\left\langle L_{k j} \varphi, \bar{\psi}\right\rangle=\left\langle\varphi, \overline{L_{k j}^{\prime} \psi}\right\rangle \text { for } \varphi, \psi \in \mathscr{Q}(\Gamma), \\
L_{\jmath k}^{\prime} \in \mathscr{L}\left(H^{-\mu_{k}^{\prime}-(1 / 2)}(\Gamma), H^{-m_{j}^{\prime}-(1 / 2)}(\Gamma)\right) .
\end{gathered}
$$

Notation 6.1. For $s \geqq 0$ we will let

$$
\begin{aligned}
\mathscr{H}_{s} & =\prod_{j=0}^{m-1} H^{s-m_{\jmath}-(1 / 2)}(\Gamma), \quad \mathfrak{A}_{s}=\prod_{j=0}^{m-1} H^{s-\mu_{j}-(1 / 2)}(\Gamma), \\
\mathscr{H}_{s}^{\prime} & =\prod_{j=0}^{m-1} H^{2 m-s-\mu_{j}^{\prime}-(1 / 2)}(\Gamma) \quad \text { and } \quad \mathfrak{A}_{s}^{\prime}=\prod_{j=0}^{m-1} H^{2 m-s-m_{j}^{\prime}-(2 / 2)}(\Gamma) .
\end{aligned}
$$

Lemia 6.2. For $u, v \in \mathscr{D}(\bar{\Omega})$ we have

$$
(A u, v)-\left(u, A^{\prime} v\right)=\sum_{j=0}^{m-1}\left\{\left\langle C_{j} u, \overline{B_{j}^{\prime} v-L_{j}^{\prime} v}\right\rangle-\left\langle B_{j} u-L_{j} u, \overline{C_{j}^{\prime} v}\right\rangle\right\} .
$$

Lemma 6.3. Let $\varphi \in \mathfrak{A}_{2 m}$. There exists $u \in V_{B-L}^{2 m}(\Omega)$ such that $C u=\varphi$, the map $\phi \rightarrow u$ being continuous.

Proof. Let $\varphi=\left(\varphi_{0}, \cdots, \varphi_{m-1}\right)$ be given and let

$$
\psi=\left(\psi_{0}, \cdots, \dot{\psi}_{m-1}\right) \in \mathscr{H}_{2 m}
$$

where $\psi_{j}=\sum_{k=0}^{m-1} L_{k \jmath} \varphi_{k}$. There is a $u \in H^{2 m}(\Omega)$ depending continuously on $(\varphi, \psi)$ such that $C u=\varphi, B u=\psi$. Then $u \in V_{B-L}^{2 m}(\Omega)$. 
THEOREM 6.4. Let $A$ be the differential operator $A$ with domain $V_{B-L}^{2 m}(\Omega)$ and $A^{*}$ the differential operator $A^{\prime}$ with domain $V_{B^{\prime}-L^{\prime}}^{0}(\Omega) \cap \mathscr{D}_{A^{\prime}}^{0}(\Omega)$. Then $A^{*}$ is the Hilbert space adjoint of $A$.

Proof. It is clear by integration by parts that $A^{*}$ is a restriction of the adjoint of $A$. If $v$ is in the domain of the adjoint of $A$ then $v \in \mathscr{D}_{A}^{0},(\Omega)$ and for $u \in V_{B-L}^{2 m}(\Omega)$

$$
\begin{aligned}
0 & =(A u, v)-\left(u, A^{\prime} v\right)=\sum_{j=0}^{m-1}\left\{\left\langle C_{j} u, \overline{B_{j}^{\prime} v}\right\rangle-\left\langle B_{j} u, \overline{C_{j}^{\prime} v}\right\rangle\right\} \\
& =\sum_{j=0}^{m-1}\left\{\left\langle C_{j} u, \overline{B_{j}^{\prime} v}\right\rangle-\left\langle L_{j} u, C_{j}^{\prime} v\right\rangle\right\} \\
& =\sum_{j=0}^{m-1}\left\langle C_{j} u, B_{j}^{\prime} v-L_{j}^{\prime} v\right\rangle .
\end{aligned}
$$

By the preceding lemma $\left(B^{\prime}-L^{\prime}\right) v=0$.

Now using the notation of the preceding theorem the operator $A^{\prime}$ with domain $V_{B^{\prime}-L^{\prime}}^{0}(\Omega) \cap \mathscr{D}_{A^{\prime}}^{0}(\Omega)$ is the adjoint of $A$. Consequently $A^{\prime *}$ the adjoint of $A^{\prime}$ with domain $V_{B^{\prime}-L^{\prime}}^{0}(\Omega)$ is $A^{* *}$. Thus

THEOREM 6.5. $A^{*}$, the Hilbert space adjoint of the operator $A^{\prime}$ with domain $V_{B^{\prime}-L^{\prime}}^{0}(\Omega) \cap \mathscr{D}_{A^{\prime}}^{0}(\Omega)$ is the closure of $A$ with domain $V_{B-L}^{2 m}(\Omega)$ and is therefore equal to $A$ with domain $V_{B-L}^{2 m}(\Omega)$ if and only if that operator is closed.

REMARK 6.6. In general $A$ with domain $V_{B-L}^{2 m}(\Omega)$ to not closed without some further restriction on $L$. For example, let $\Omega=\{(x, t)$ : $x \in R^{n}$ and $\left.t>0\right\}$ so that $\Gamma=R^{n}$. Let $\Delta=\sum_{j=1}^{n} D_{j}^{2}$ and $I$ be the identity map of $L^{2}(\Omega)$ onto $L^{2}(\Omega)$. Let $\xi$ denote the dual variable to $x$ and $\hat{f}$ the Fourier-Plancherel transform of $f$ in $L^{2}(I)$. Define $L \in \mathscr{L}\left(H^{1 / 2}(\Gamma), H^{1 / 2}(\Gamma)\right)$ by $(L f)^{\wedge}(\xi)=-\left(1+|\xi|^{2}\right)^{1 / 2} \hat{f}(\xi)$ so that $L$ is in fact an isomorphism. Let the domain of $A$ be $\left\{u \in H^{2 m}(\Omega):(\partial u / \partial t)-L u=0\right.$ on $\Gamma\}$ and for such $u$ let $A u=(I+\Delta) u$. If $u$ is such that $\widehat{u}(\xi, t)=$ $\exp \left\{-\left(1+|\xi|^{2}\right)^{1 / 2} t\right\} \hat{f}(\xi)$ where $f \in H^{3 / 2}(\Gamma)$ then $u$ is the domain of $A$ but there is no $C>0$ such that $\|u\|_{2 m} \leqq C\left(\|(I+\Delta) u\|_{0}+\|u\|_{0}\right)$ for all such $u$. By Proposition 4.7, $A$ cannot be closed.

In view of the inequality 4.1 it is easy to impose a norm condition on $L$ in order that $A$ with domain $V_{B \rightarrow L}^{2 m}(\Omega)$ be closed.

THEOREM 6.7. If there exists a $\delta: 0<\delta<1 / m$ such that for $0 \leqq j \leqq m-1$ and all $u \in H^{2 m}(\Omega)$

$$
\left\|L_{j} u\right\|_{2 m-m_{j}-(1 / 2)} \leqq \delta / C_{2 m}\|u\|_{2 m}+K\left[\|A u\|_{0}+\|u\|_{0}\right],
$$

then $A$ with domain $V_{3-L}^{2 m}(\Omega)$ is closed. 
For the remainder of this section we assume that the operator $A_{B}$ has closed range so that by Corollary 5.15 for all $u \in N_{2 m, B}^{\perp}$

$$
\|u\|_{2 m} \leqq C_{2 m}^{\prime}\left[\|A u\|_{0}+\sum_{j=0}^{m-1}\left\|B_{j} u\right\|_{2 m-m_{j}-(1 / 2)}\right] .
$$

Since the Hilbert space adjoint of $A_{B}$ is $A_{B^{\prime}}^{\prime}$ it also has closed range so that for $u \in N_{2 m}^{\perp}, B^{\prime}$

$$
\|u\|_{2 m} \leqq C_{2 m}^{\prime}\left[i \mid A^{\prime} u\left\|_{0}+\sum_{j=0}^{m-1}\right\| B_{j}^{\prime} u \|_{2 m-m_{j}^{\prime}-(12)}\right]
$$

where the constants $C_{2 m}^{\prime}$ may not be the same. Thus we can immediately assert

Theorem 6.8. Suppose $L \in \mathscr{L}\left(H^{2 m}(\Omega), \mathscr{H}_{2 m}\right)$ and that there exists a $\delta: 0<\delta<1 / m$ and such that for $0 \leqq j \leqq m-1$ and $u \in H^{2 m}(\Omega)$ we have $\left\|L_{j} u\right\|_{2 m-m_{j}-(1 / 2)} \leqq \delta / C_{2 m}^{\prime}\|u\|_{2 m}$. Then for $v \in N_{2 m, B}^{\perp}$ we have

$$
\|u\|_{2 m} \leqq K_{2 m}^{\prime}\left[\|A u\|_{0}+\sum_{j=0}^{m-1}\left\|\left(B_{j}-L_{j}\right) u\right\|_{2 m-m_{j}-(1 / 2)}\right] .
$$

Thus the operator $A$ with domain $V_{B-L}^{2 m}(\Omega)$ also has closed range and its kernel is contained in $N_{B}$. In particular if $A_{B}$ is injective then $A$ with domain $V_{B-L}^{2 m}(\Omega)$ is also injective and has a continuous inverse.

For $u \in \mathscr{D}_{A^{\prime}}^{0}(\Omega) \cap N_{B^{\prime}, 0}^{\perp}$ we have by Corollary 5.19,

$$
\|u\|_{0} \leqq C_{0}^{\prime}\left[\left\|A^{\prime} u\right\|_{0}+\sum_{j=0}^{m-1}\left\|B_{\jmath}^{\prime} u\right\|_{-m_{j}^{\prime}-(1 / 2)}\right] .
$$

Now let $K>0$ be such that for $u \in H^{2 m}(\Omega)$ : $\left\|C_{\jmath} u\right\|_{2 m-\mu_{j}-(1 / 2)} \leqq K\|u\|_{2 m}$, $0 \leqq j \leqq m-1$ and for $u \in \mathscr{D}_{A^{\prime}}^{0}(\Omega),\left\|C_{j}^{\prime} u\right\|_{-\mu_{j}^{\prime}-(1 / 2)} \leqq K\left[\|u\|_{0}+\|A u\|_{0}\right]$. Let $0<\delta<1 / m$ and suppose $\sum_{l k=0}^{m-1}\left\|L_{k j}\right\| \leqq \min \left[\delta / m K C_{0}^{\prime}, \delta / m K C_{2 m}^{\prime}\right]$ for $0 \leqq j \leqq m-1$ and that $\sum_{k=0}^{m-1}\left\|L_{j k}^{\prime}\right\| \leqq \min \left[\delta / m K C_{0}^{\prime}, \delta / m K C_{2 m}^{\prime}\right]$. Then for $u \in \mathscr{D}_{{ }^{\prime}}^{0},(\Omega) \cap N_{B^{\prime}, 0}^{\perp}$ we have

$$
\|u\|_{0} \leqq C_{0}^{\prime}\left[\left\|A^{\prime} u\right\|_{0}+\sum_{j=0}^{m-1}\left\|\left(B_{j}^{\prime}-L_{j}^{\prime}\right) u\right\|_{m_{j}^{\prime}-(1 / 2)}\right] .
$$

THEOREM 6.9. Suppose that $A_{B}$ is an isomorphism and that $L_{j}, L_{j}^{\prime}, 0 \leqq j \leqq m-1$ satisfy the above conditions. Then for $u \in H^{2 m}(\Omega)$

$$
\|u\|_{2 m} \leqq C_{2 m}^{\prime}\left[\|A u\|_{0}+\sum_{j=0}^{m-1}\left\|\left(B_{j}-L_{j}\right) u\right\|_{2 m-m_{j}-(1 / 2)}\right]
$$

and for $u \in \mathscr{D}_{A^{\prime}}^{0}(\Omega)$

$$
\|u\|_{0} \leqq C_{0}^{\prime}\left[\left\|A^{\prime} u\right\|_{0}+\sum_{j=0}^{m-1}\left\|\left(B_{j}^{\prime}-L_{j}^{\prime}\right) u\right\|_{-m_{j}^{\prime}-(1 / 2)}\right] .
$$


Thus $A$ and its adjoint are isomorphisms on their respective domains.

Corollary 6.10. Suppose that in addition to the hypothesis of Theorem $6.9 \mathrm{~L}$ can be extended to $\mathscr{D}_{A}^{0}(\Omega)$ so as to be a continuous linear map of $\mathscr{D}_{A}^{0}(\Omega)$ into $\mathscr{H}_{0}$ and that there exists a $\delta: 0<\delta<1 / m$ such that for $0 \leqq j \leqq m-1 \quad$ and $u \in \mathscr{D}_{A}^{0}(\Omega) \quad\left\|L_{\jmath} u\right\|_{-m_{j}-(1 / 2)} \leqq$ $\delta / C_{0}\left[\|u\|_{0}+K\|A u\|_{-2 m}\right]$ where $\|\cdot\|_{-2 m}$ is the norm in $H_{\overline{\bar{\Omega}}}^{2 m}$. Then the domain of $A^{*}$, where domain of $A$ is $V_{B-L}^{2 m}(\Omega)$, is contained in $H^{2 m}(\Omega)$.

Proof. Using the condition on $L$ and Theorem 5.23 we have

$$
\|u\|_{0} \leqq K\left[\|A u\|_{-2 m}+\sum_{j=0}^{m-1}\left\|\left(B_{j}-L_{j}\right) u\right\|_{-m_{j}-(1 / 2)}\right]
$$

for $u \in \mathscr{D}_{A}^{0}(\Omega)$ and thus for $u \in \mathscr{D}_{A}^{0}(\Omega) \subset V_{B-L}^{0}(\Omega)$ we have $\|u\|_{0} \leqq$ $K\|A u\|_{-2 m}$. Let $v \in$ domain of the adjoint of $A$. Then there exists a number $k(v)>0$ such that for $u \in V_{B \rightarrow L}^{2 m}(\Omega),\left|(A u, v)_{0}\right| \leqq k(v)\|u\|_{0} \leqq$ $K k(v)\|A u\|_{-2 m}$. The linear form $F$ on the image of $A$ defined by $F(A u)=(A u, v)_{0}$ is thus continuous in $H_{\overline{\bar{l}}}^{2 m}$ and can be extended so as to be continuous in all of $H_{\overline{\bar{\Omega}}}^{2 m}$. There exists a $w \in H^{2 m}(\Omega)$ such that $F(\cdot)=(\cdot, w)_{0}$. It follows that $(A u, v)_{0}=(A u, w)_{0}$ or that $(A u, v-w)_{0}=0$ for $u \in V_{B-L}^{2 m}(\Omega)$. But since $A$ is an isomorphism on $V_{B-L}^{2 m}(\Omega), v=w \in H^{2 m}(\Omega)$.

Proposition 6.11. If each $L_{j}$ is compact then $A$ with domain $V_{B-L}^{2 m}(\Omega)$ has closed range.

Proof. The map $u \rightarrow(A u, B u)$ has closed range in $H^{0}(\Omega) \times \mathscr{H}_{2 m}$ and the map $u \rightarrow(0,-L u)$ is compact. Thus the map $u \rightarrow(A u, B u-L u)$ has closed range and hence the map $u \rightarrow A u$ of $V_{B-L}^{2 m}(\Omega) \rightarrow L^{2}(\Omega)$ has closed range.

Proposition 6.12. Suppose $\Omega$ is relatively compact and that $A$ with domain $V_{B-L}^{2 m}(\Omega)$ is closed. Then the kernel of $A$ is finite dimensional and the resolvent of $A$, when it exists, is compact. The range of $A$ is closed. If the domain of $A^{*}$ is contained in $\mathscr{D}_{A}^{s}(\Omega)$ with $s>0$ then the range of $A$ has finite codimension.

Proof. The proof of each statement but the last is no different from the usual proof for operators defined by differential boundary conditions. As for the last statement since the domain of $A^{*}$ is contained in $H^{s}(\Omega)$ then by the closed graph theorem

$$
\|u\|_{s} \leqq C_{s}^{\prime}\left[\left\|A^{\prime} u\right\|_{0}+\|u\|_{0}\right]
$$


for $u \in \mathscr{D}\left(A^{*}\right)$. It follows from Rellich's lemma that the kernel of $A^{*}$ is finite dimensional and thus that the codimension of the range of $A$ is finite.

\section{Some consequences of interpolation.}

\section{DEFINITION 7.1.}

$$
\tilde{\mathscr{D}}^{s}(A, B-L, \Omega)=V_{B-L}^{s}(\Omega) \cap \tilde{\mathscr{D}}_{A}^{s}(\Omega)
$$

where $L \in \mathscr{L}\left(H^{s}(\Omega), \mathscr{H}_{s}\right)$

We will extend the method used in Bade and Freeman [3] to higher order equations. In the remainder of this section we assume that the operator $A_{B}$ has closed range.

Theorem 7.2. Suppose $L \in \mathscr{L}\left(H^{s}(\Omega), \mathscr{H}_{s}\right)$. Then for $0 \leqq s \leqq 2 m$

(i) If for $u \in \tilde{\mathscr{D}}_{A}^{s}(\Omega)$ and $0 \leqq j \leqq m-1,\left\|L_{j} u\right\|_{s-m_{j-(1 / 2)}} \leqq$ $\delta / C_{s}\|u\|_{s}+K_{s}\left[\|A u\|_{0}+\|u\|_{0}\right]$ where $0<\delta<1 / m$ then the operator $A$ with domain $\tilde{\mathscr{D}}^{s}(A, B-L, \Omega)$ has closed graph;

(ii) if for $u \in \tilde{\mathscr{D}}_{A}^{s}(\Omega)$ and $0 \leqq j \leqq m-1,\left\|L_{j} v\right\|_{s-m_{j}-(1 / 2)} \leqq$ $\delta / C_{s}^{\prime}\|v\|_{s}+K_{s}\|A u\|_{0}$ where $0<\delta<1 / m$ then the operator $A$ with domain $\tilde{\mathscr{D}}^{s}(A, B-L, \Omega)$ is closed and has closed range. Its kernel is contained in $N_{B}$.

Proof. By (i) and (5.5) we have for $u \in \tilde{\mathscr{D}}_{A}^{s}(\Omega)$

$$
\|u\|_{s} \leqq C_{s}\left[\|A u\|_{0}+\|u\|_{0}+\sum_{j=0}^{m-1}\left\|\left(B_{j}-L_{j}\right) u\right\|_{s-m_{j}-(1 / 2)}\right]
$$

which implies the result.

By (ii) and (5.4) we have for $u \in \tilde{\mathscr{C}}_{A}^{s}(\Omega) \cap N_{s_{1} B}^{\perp}$

$$
\|u\|_{s} \leqq C_{s}^{\prime}\left[\|A u\|_{0}+\sum_{j=0}^{m-1}\left\|\left(B_{j}-L_{j}\right) u\right\|_{s-m_{j}-(1 / 2)}\right] .
$$

For $u \in \tilde{\mathscr{D}}_{A}^{s}(\Omega) \quad u=u^{\prime}+u^{\prime \prime}$ where $u^{\prime} \in N_{B}$ and $u^{\prime \prime} \in \tilde{\mathscr{D}}_{A}^{s}(\Omega) \cap N_{s_{1} B}^{\perp}$. Since for $u^{\prime} \in N_{B}, A u^{\prime}=0$ we have $\left\|\left(B_{j}-L_{j}\right) u^{\prime}\right\|_{s-m_{j}-(1 / 2)} \leqq K\left\|u^{\prime}\right\|_{s}$. Moreover $\left\|u^{\prime}\right\|_{s} \leqq K\|u\|_{0}$ where here $K$ represents a constant, not necessarily the same one each time it appears. Thus writing

$$
\begin{aligned}
\|u\|_{s} \leqq\left\|u^{\prime}\right\|_{s} & +\left\|u^{\prime \prime}\right\|_{s} \leqq K\|u\|_{0} \\
& +C_{s}^{\prime}\left[\|A u\|_{0}+\sum_{j=0}^{m-1}\left\|\left(B_{j}-L_{j}\right) u\right\|_{s-m j-(1 / 2)}+K^{\prime}\|u\|_{0}\right]
\end{aligned}
$$

yields 


$$
\|u\|_{s} \leqq C_{s}\left[\|A u\|_{0}+\|u\|_{0}+\sum_{j=0}^{m-1}\left\|\left(B_{j}-L_{j}\right) u\right\|_{s-m_{j}-(1 / 2)}\right] .
$$

This coupled with (7.1) yields (ii).

REMARK 7.3. Analogous results are valid for $A^{\prime}, B^{\prime}-L^{\prime}$.

Following the procedure used in Bade and Freeman [3] we define a map on $H^{\circ}(\Omega) \times \mathfrak{A}_{s}$ as follows.

Definition 7.4. Let the domain of $S$ be the set of $(u, C u)$ such that $u \in \tilde{\mathscr{D}}_{A}^{s}(\Omega)$ and let $S(u, C u)=(A u, B u)$. Thus $S: H^{0}(\Omega) \times \mathfrak{Y}_{s} \rightarrow$ $H^{0}(\Omega) \times \mathscr{H}_{s}$. Analogously let the domain of $S^{\prime}$ be the set of $\left(u, C^{\prime} u\right)$ such that $u \in \tilde{\mathscr{D}}_{A^{\prime}}^{2 m-s}(\Omega)$ and let $S^{\prime}\left(u, C^{\prime} u\right)=\left(A^{\prime} u, B^{\prime} u\right)$. Thus $S^{\prime}: H^{0}(\Omega) \times$ $\mathfrak{U}_{s}^{\prime} \rightarrow H^{0}(\Omega) \times \mathscr{H}_{s}^{\prime}$.

TheOREM 7.5. $S\left(S^{\prime}\right)$ is closed and densely defined. The adjoint of $S\left(S^{\prime}\right)$ is $S^{\prime}(S)$.

Proof. We prove the result for $S$, the proof for $S^{\prime}$ being identical modulo a change of notation. Closedness follows immediately by (5.6) of Theorem 5.20 and the fact that $C$ is continuous on $\tilde{\mathscr{D}}_{A}^{s}(\Omega)$. That the domain of $S$ is dense in $H^{\circ}(\Omega) \times \mathfrak{A}_{s}$ is clear.

Suppose

$$
(v, \varphi,-f, \psi) \in H^{0}(\Omega) \times \mathfrak{X}_{0}^{\prime} \times H^{0}(\Omega) \times \mathscr{H}_{s}^{\prime}
$$

and for $u \in \tilde{\mathscr{D}}_{A}^{s}(\Omega)$

$$
(A u, v)+\sum_{j=0}^{m-1}\left\langle B_{\jmath} u, \overline{\varphi_{j}}\right\rangle-(u, f)-\sum_{j=0}^{m-1}\left\langle C_{j} u, \overline{\psi_{j}}\right\rangle=0 .
$$

Then for $u \in N_{B}$ we have $-(u, f)-\sum_{j=0}^{m-1}\left\langle C_{j} u, \overline{\psi_{j}}\right\rangle=0$ and applying part (ii) of Theorem 5.20 to $A^{\prime}, B^{\prime}, C^{\prime}$ and $\tilde{\mathscr{D}}_{A^{\prime}}^{2 m-s}(\Omega)$ there exists $w \in \tilde{\mathscr{D}}_{A^{\prime}}^{2 m-s}(\Omega)$ such that $A^{\prime} w=f$ and $B^{\prime} w=\psi$. But then for $u \in \tilde{\mathscr{D}}_{A}^{s}(\Omega)$ we have

$$
\begin{aligned}
(A u, w)-\left(u, A^{\prime} w\right) & =\sum_{j=0}^{m-1}\left\{\left\langle C_{j} u, \overline{B_{j}^{\prime} w}\right\rangle-\left\langle B_{j} u, \overline{C_{j}^{\prime} w}\right\rangle\right\} \\
= & (A u, w)-(u, f)-\sum_{j=0}^{m-1}\left\{\left\langle C_{j} u, \overline{\psi_{j}}\right\rangle-\left\langle B_{j} u, \overline{C_{j}^{\prime} w}\right\rangle\right\}=0 .
\end{aligned}
$$

Combining this with (7.3) yields for $u \in \tilde{\mathscr{D}}_{A}^{s}(\Omega)$

$$
(A u, v-w)+\sum_{j=0}^{m-1}\left\langle B_{j} u, \overline{\varphi_{j}-C_{j}^{\prime \prime} w}\right\rangle=0 .
$$

In particular for $u \in V_{B}^{2 m}(\Omega)$ we have $(A u, v-w)=0$ and thus 
$v-w \in N_{B^{\prime}} \subset H^{2 m}(\Omega)$. Thus $v=w+(v-w) \in \tilde{\mathscr{D}}_{A^{\prime}}^{2 m-s}(\Omega)$ and $B^{\prime} v=$ $B^{\prime} w=\psi^{\prime}$ and $A^{\prime} v=A^{\prime} w=f$. Thus we have for $u \in \tilde{\mathscr{D}}_{A}^{s}(\Omega)$

$$
(A u, v)-(u, f)-\sum_{j=0}^{m-1}\left\{\left\langle C_{j} u, \overline{\psi_{j}}\right\rangle-\left\langle B_{j} u, \overline{C_{j}^{\prime} v}\right\rangle\right\}=0 .
$$

Combining this with (7.3) yields $\sum_{i j=0}^{m-1}\left\langle B_{j} u, \overline{\varphi_{j}-C_{j}^{\prime \prime} v}\right\rangle=0$ for $u \in \tilde{\mathscr{D}}_{A}^{s}(\Omega)$ which implies that $\varphi_{j}-C_{j}^{\prime} v=0$ for $j=0, \cdots, m-1$.

THEOREM 7.6. Let $L \in \mathscr{L}\left(H^{s}(\Omega), \mathscr{H}_{s}\right)$ and define $\hat{L}$ by $\hat{L}(u, C u)=$ $(0, L C u)$. Then $\hat{L} \in \mathscr{L}\left(H^{0}(\Omega) \times H^{s}(\Omega), H^{0}(\Omega) \times \mathscr{C}_{s}\right)$ and the dual of $S-\hat{L}$ is $S^{\prime}-\hat{L}^{\prime}$. Moreover, if $L$ satisfies the conditions of Theorem 7.2, part (ii), $S-\hat{L}$ has closed range in $H^{0}(\Omega) \times \mathscr{H}_{s}$.

We can now apply the procedure of Bade and Freeman [3], in particular Lemmas 5.8-5.10 to show the following

THEOREM 7.7. With the above hypotheses on $L$ and an analogous one on $L^{\prime}$ the operator $A$ with domain $\tilde{\mathscr{D}}^{s}(A, B-L, \Omega)$ is a closed, densely defined linear operator in $L^{2}(\Omega)$, with closed range. Its adjoint operator is $A^{\prime}$ with domain $\tilde{\mathscr{D}}^{2 m-s}\left(A^{\prime}, B^{\prime}-L^{\prime}, \Omega\right)$ which is also closed and has closed range. Moreover, $(f, \varphi) \in H^{\circ}(\Omega) \times \mathscr{H}_{s}$ is in the image of $(A, B-L)$ on $\tilde{\mathscr{D}}_{A}^{s}(\Omega)$ if and only if for all $v$ in the kernel of $A^{\prime}$ on $\tilde{\mathscr{D}}^{2 m-s}\left(A^{\prime}, B^{\prime}-L^{\prime}, \Omega\right)$ we have

$$
(f, v)-\sum_{j=0}^{m-1}\left\langle\varphi_{j}, \overline{\left(B_{j}^{\prime}-L_{j}^{\prime}\right) v}\right\rangle=0 .
$$

Finally if $A_{B}$ is an isomorphism so is $A$ with domain $\tilde{\mathscr{D}}^{s}(A, B-L, \Omega)$.

REMARK. Most of this has already been proved.

Corollary 7.8. If $\Omega$ is relatively compact and $0<s<2 m$ the operator $A$ with domain $\tilde{\mathscr{D}}^{s}(A, B-L, \Omega)$ has finite dimensional kernel. Its range is closed and has finite codimension. The resolvent operator where it exists is compact.

REMARK 7.9. The example of Remark 6.6 works just as well in $\mathscr{D}_{A}^{s}(\Omega)$ for $0<s<2$ to show that some additional condition (other than continuity) is required of $L$.

8. Lower bound conditions. Let $a(u, v)=\sum_{|\alpha|,|\beta| \leq m}\left(a_{\alpha \beta} D^{\alpha} u, D^{\beta} v\right)$. Then we can write

$$
(A u, u)=a(u, v)+\sum_{j=0}^{m-1} \int_{\Gamma} N_{j} u \overline{\gamma_{j} v} d \sigma
$$


where $\left\{N_{j}: 0 \leqq j \leqq m-1\right\}$ is a normal set of boundary operators with infinitely differentiable coefficients, the order of $N_{j}$ being $2 m-j-1$. The system $(A, N)$ is closable elliptic. Similarly

$$
\left(u, A^{\prime} v\right)=a(u, v)+\sum_{j=0}^{m-1} \int_{\Gamma} \gamma_{j} u \overline{N_{j}^{\prime} v} d \sigma .
$$

Let $N\left(N^{\prime}\right)$ denote the set $\left\{N_{j}: 0 \leqq j \leqq m-1\right\}\left(\left\{N_{j}^{\prime}: 0 \leqq j \leqq m-1\right\}\right)$. If $C\left(C^{\prime}\right)$ is a Dirichlet set of order $m, C=\left\{C_{0}, \cdots, C_{m-1}\right\}, C^{\prime}=$ $\left\{C_{0}^{\prime}, \cdots, C_{m-1}^{\prime}\right\}$, the order of $C_{j}\left(C_{j}^{\prime}\right)$ being $j$, we can find another normal set $B=\left\{B_{0}, \cdots, B_{m-1}\right\}$ and $B^{\prime}=\left\{B^{\prime}, \cdots, B_{m-1}^{\prime}\right\}$, the order of $B_{j}\left(B_{j}^{\prime}\right)$ being $2 m-j-1$. The above formula remains valid if $N_{j}, \gamma_{j}, N_{j}^{\prime}, \gamma_{j}$ are replaced by $B_{j}, C_{j}^{\prime}, B_{j}^{\prime}, C_{j}$.

We use the terminology of Lions-Magenes [16] and say

Definition 8.1. $A$ is $H^{m}(\Omega)$ elliptic if and only if for all $u \in H^{m}(\bar{\Omega})$ $\operatorname{Re} a(u, u) \geqq \alpha_{0}\|u\|_{m}^{2}, \alpha_{0}$ some positive real number.

Let $L_{j} \leqq \sum_{i k=0}^{m-1} L_{k j} \gamma_{k}$ where $L_{k j} \in \mathscr{P}\left(H^{m-k-(1 / 2)}(\Gamma), H^{-m+j+(1 / 2)}(\Gamma)\right)$ and $L=\left(L_{0}, \cdots, L_{m-1}\right)$. Then $L_{j} \in \mathscr{P}\left(H^{m}(\Omega), H^{-m+j+\{1 / 2\rangle}(\Gamma)\right)$ and we will write $L \in \mathscr{P}\left(\prod_{j=0}^{m-1} H^{m}(\Omega), \prod_{\jmath=0}^{m-1} H^{-m+j+(1 / 2)}(\Gamma)\right)$. Let

$$
a_{L}(u, v)=a(u, v)+\sum_{j=0}^{m-1}\left\langle L_{j} u, \overline{\gamma_{j} v}\right\rangle
$$

for $u, v \in H^{m}(\Omega)$.

Proposition 8.2. $a_{L}(\cdot, \cdot)$ is a continuous sesquilinear form on $H^{m}(\Omega)$ and defines a linear map $A$ of a dense subspace of $H^{0}(\Omega)$ into $H^{0}(\Omega)$.

REMARK 8.3. The linear map does not necessarily have closed graph. See the example of Remark 7.9. If additional conditions are imposed on $L$ so that $\operatorname{Re} a_{L}(u, u) \geqq \alpha_{1}\|u\|_{m}^{2}$ then the operator is not only closed but is an isomorphism of its domain with the graph topology onto $H^{\circ}(\Omega)$. This is essentially the result of Lax and Milgram [12]; see also Freeman [9], Theorem 2.2. Some conditions which guarantee this are stated in the following

THEOREM 8.4. Suppose $A$ is $H^{m}(\Omega)$ elliptic and that $a_{L}$ is given by Definition 8.1. Suppose

(i) $\operatorname{Re} \sum_{j=0}^{m-1}\left\langle L_{j} u, \overline{\gamma_{j} u}\right\rangle \geqq \alpha_{1}\|u\|_{m}^{2}$ with $\alpha_{1}+\alpha_{0}>0$. Then the operator $A$ given by Proposition 8.2 is an isomorphism.

(ii) If $1 / 2<s_{\jmath}<1$ and for $0 \leqq j \leqq m-1, L_{j} \in \mathscr{P}\left(H^{m}(\Omega)\right.$, $\left.H^{-\left(m-j-s_{j}\right)}(I)\right)$ or $L_{j} \in \mathscr{L}\left(H^{m-s_{j}}(\Omega), H^{-m+j+(1 / 2)}(\Gamma)\right)$ then (i) is valid for $a_{L}(u, v)+\lambda(u, v)$ where $\lambda>0$ is sufficiently large, i.e. thus the operator 
$A+\lambda I$ is an isomorphism.

Proof. Part (i) is immediate. For Part (ii) we have either

$$
\left|\left\langle L_{j} u, \overline{\gamma_{j} u}\right\rangle\right| \leqq K_{j}\|u\|_{m}\left\|\gamma_{j} u\right\|_{m-j-s_{j}}
$$

or

$$
\left|\left\langle L_{j} u, \overline{\gamma_{j} u}\right\rangle\right| \leqq K_{j}\|u\|_{m-s_{j}}\left\|\gamma_{j} u\right\|_{m-j-(1 / 2)} \leqq K_{j}\|u\|_{m-s_{j}}\|u\|_{m} .
$$

Then using either Lemma 3.6 and Lemma 3.1 or Lemma 2.12 we find that given $\varepsilon>0$ there exists a $C(\varepsilon)>0$ such that $\sum\left|\left\langle L_{j} u, \overline{\gamma_{j} u}\right\rangle\right| \leqq$ $\varepsilon\|u\|_{m}^{2}+C(\varepsilon)\|u\|_{0}^{2}$ and (ii) follows from (i) by choosing $\varepsilon>0$ sufficiently small.

COROLlaRY 8.5. Under the above conditions and for the same $\lambda, A^{\prime}+\lambda I$ is also an isomorphism. The operator $A\left(A^{\prime}\right)$ has domain $\mathscr{D}^{m}(A, N-L, \Omega)\left(\mathscr{D}^{m}\left(A^{\prime}, N^{\prime}-L^{\prime}, \Omega\right)\right)$.

Corollary 8.6. The adjoint of $A$ with domain $\mathscr{D}^{m}(A, N-L, \Omega)$ is $A^{\prime}$ with domain $\mathscr{D}^{m}\left(A^{\prime}, N^{\prime}-L^{\prime}, \Omega\right)$.

REMARK 8.7. If Corollary 8.6 were true for any $L \in \mathscr{L}\left(\prod_{j=0}^{m-1} H^{m}(\Omega)\right.$, $\left.\Pi_{j=0}^{m-1} H^{-(m-j-(1 / 2))}(\Gamma)\right)$ with no additional conditions required then it would also be true that $A$ with domain $\mathscr{D}^{m}(A, N-L, \Omega)$ is the adjoint of $A^{\prime}$ with domain $\mathscr{D}^{m}\left(A^{\prime}, N^{\prime}-L^{\prime}, \Omega\right)$ and would thus be closed. Thus by Remark 8.3 some additional condition on $L$ is needed. If $N_{j}, \gamma_{j}, N_{j}^{\prime}, \gamma_{j}$ are replaced by $B_{j}, C_{j}^{\prime}, B_{j}^{\prime}, C_{j}$ the result is still true.

After this manuscript was completed we learned that Schechter has considered similar problems in $L^{p}$ but with bounded $\Omega$. The boundedness of $\Omega$ is essential because he needs Rellich's lemma. In this manuscript which he kindly sent us he has an elegant proof of the inequality

$$
\left\|B_{j} u\right\|_{s-m_{j}-(1 / 2)} \leqq c\left[\|A u\|_{s-2 m}+\|u\|_{s}\right] \text { for } u \in(\bar{\Omega}) .
$$

It follows from this that $\tilde{\mathscr{D}}_{A}^{s}(\Omega)$ is the completion in the norm $\left(\|\cdot\|_{s}^{2}+\|A\|_{0}^{2}\right)^{1 / 2}$ of $\mathscr{D}(\bar{\Omega})$ under the closed range assumption.

We wish at this time to express our gratitude to the referee for his comments and suggestions. In particular Theorem 8.4 is basically his generalization of our result and the proof is basically his. Also a question of his is answered in the negative by Remark 8.7. 


\section{BIBLIOGRAPHY}

1. S. Agmon, A. Douglis and L. Nirenberg, Estimates near the boundary of solutions of elliptic partial differential equations satisfying general boundary conditions I, Comm. Pure Appl. Math. 12 (1959), 623-727.

2. N. Aronszajn and A. N. Milgram, Differential operators on Riemann manifolds, Rend. Circ. Mat. Palermo (2) (1953), 266-325.

3. W. G. Bade and R. S. Freeman, Closed extensions of the Laplace operator determined by a general class of boundary conditions, Pacific J. Math. 12 (1962), 395-410.

4. R. W. Beals, Non-local boundary value problems for elliptic partial differential operators, Thesis, Yale University, 1964.

5. F. E. Browder, Estimates and existence theorems for elliptic boundary value problems, Proc. Nat. Acad. Sci. 45 (1959), 365-372.

6. - On the spectral theory of elliptic differential operators I, Math. Ann. 142 (1961), 22-130.

7. A. P. Calderon, Intermediate spaces and interpolation, the complex method, Studia Math. 24 (1964), 113-190.

8. G. Ehrling, On a type of eigenvalue problem for certain elliptic differential operators, Math. Scand. 2 (1954), 267-285.

9. R. S. Freeman, Closed extensions of the Laplace operator determined by a general class of boundary conditions for unbounded regions, Pacific J. Math. 12 (1962), 121-135. 10. - Some remarks on boundary problems, Proc. Amer. Math. Soc. 16 (1965), $1253-1255$.

11. T. Kato, Perturbation theory for nullity, deficiency and other quantities of linear operators, J. d'Analyse Math. (1958), 261-322.

12. P. D. Lax and A. N. Milgram, Parabolic equations, Contributions to the Theory of Partial Differential Equations, Ann. of Math. Studies, No. 33, Princeton University Press (1954), 167-190.

13. J. L. Lions, Lectures on partial differential equations, Tata Institute lecture notes, Bombay, 1957.

14. Une construction d'espace d'interpolation, Compte Rendue Acad. Sci., Paris 244 (1959), 389-403.

15. - Espace intermédiares entre espaces hilbertiens et applications, Bull. Math. Soc. Sci. Math. Phys. Roumanie 50 (1958), 419-432.

16. J. L. Lions and E. Magenes, Problèmes aux limites non homogenes (II), Ann. Inst. Fourier 11 (1961), 137-178.

17. - Problemi ai limite non omogenii (III), Ann. Scuola Norm. Sup. Pisa 15 (1961), 39-101.

18. - Problèmes aux limites non homogenes (IV), Ann. Scuola Norm. Sup. Pisa 15 (1961), 311-326.

19. - Problemi ai limite non omogenii (V), Ann. Scuola Norm. Sup. Pisa 16 (1962), 1-44.

20. J. L. Lions and J. Peetre, Sur une classe d'espaces d'interpolation, Inst. des hautes études scientifiques, Publications Mathématiques, No. 19 (1964), 5-68.

21. E. Magenes and G. Stampacchia, I problemi al contorno per le equazione differenziale di tipe ellittico, Aun. Scuola Norm. Sup. Pisa, II 12 (1958), 247-357.

22. J. Peetre, Another approach to elliptic boundary problems, Comm. Pure Appl. Math. 14 (1961), 711-731.

23. M. Schechter, Integral inequalities for partial differential operators and functions satisfying general boundary conditions, Comm. Pure Appl. Math. 12 (1959), 37-66.

24. - General boundary value problems for elliptic partial differential equations, Comm. Pure Appl. Math. 12 (1959), 457-486.

25. - On $L^{p}$ estimates and regularity II, Math. Scand. 13 (1963), 47-69.

26. - Negative norms and boundary problems Ann. of Math. (2) 72 (1960), 
581-593.

27. L. Schwartz, Théorie des distributions, Hermann et Cie, Paris, 1950.

28. M. I. Visik, On general boundary value problems for elliptic partial differential equations, Trudi Mosk. Mat. Obshch 1 (1952), 187-246; Amer. Math. Soc. Translations (2), 24, 107-172.

Received November 27, 1961. The preparation of this paper was supported by NSF Grant No. NSF GP1834. 



\section{PACIFIC JOURNAL OF MATHEMATICS}

\section{EDITORS}

\section{H. SAMELSON}

Stanford University

Stanford, California

J. P. JANS

University of Washington

Seattle, Washington 98105
J. Dugundji

University of Southern California Los Angeles, California 90007

RICHARD ARENS

University of California

Los Angeles, California 90024

\section{ASSOCIATE EDITORS}
E. F. BECKENBACH
B. H. NeumanN
F. WOLF
K. YosidA

\section{SUPPORTING INSTITUTIONS}

UNIVERSITY OF BRITISH COLUMBIA

CALIFORNIA INSTITUTE OF TECHNOLOGY

UNIVERSITY OF CALIFORNIA

MONTANA STATE UNIVERSITY

UNIVERSITY OF NEVADA

NEW MEXICO STATE UNIVERSITY

OREGON STATE UNIVERSITY

UNIVERSITY OF OREGON

OSAKA UNIVERSITY

UNIVERSITY OF SOUTHERN CALIFORNIA
STANFORD UNIVERSITY

UNIVERSITY OF TOKYO

UNIVERSITY OF UTAH

WASHINGTON STATE UNIVERSITY

UNIVERSITY OF WASHINGTON

AMERICAN MATHEMATICAL SOCIETY CHEVRON RESEARCH CORPORATION TRW SYSTEMS

NAVAL ORDNANCE TEST STATION 


\section{Pacific Journal of Mathematics \\ Vol. 22, No. $1 \quad$ January, 1967}

Charles A. Akemann, Some mapping properties of the group algebras of a

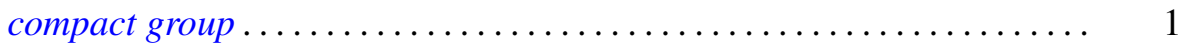

A. V. Boyd, Note on a paper by Uppuluri ..................... 9

Thomas Craig Brown, A semigroup union of disjoint locally finite subsemigroups which is not locally finite .....................

Richard Thomas Bumby and Everett C. Dade, Remark on a problem of

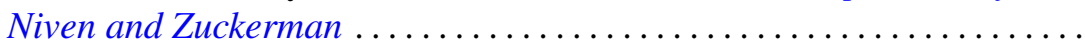

James Calvert, An integral inequality with applications to the Dirichlet problem .........................................

Jack Gary Ceder and Terrance Laverne Pearson, On products of maximally resolvable spaces ....................................

William Guignard Faris, The product formula for semigroups defined by

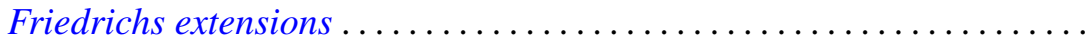

Robert S. Freeman, Closed operators and their adjoints associated with elliptic differential operators ........................ 71

Thomas Lee Hayden, The extension of bilinear functionals ............. 99

Gloria Conyers Hewitt, Limits in certain classes of abstract algebras . . . . . 109

Tilla Weinstein, The dilatation of some standard mappings ........... 117

Mitsuru Nakai, On Evans' kernel ......................... 125

Ernest Levane Roetman, On the biharmonic wave equation ............ 139

Malcolm Jay Sherman, Operators and inner functions ... . .

Walter Laws Smith, On the weak law of large numbers and the generalized elementary renewal theorem

A. J. Ward, On H-equivalence of uniformities: The Isbell-Smith problem 